\title{
Pascal Millet
}

Médecin hospitalier, et professeur, Programme de formation EPSSEL

[Éducation et Promotion Santé et Social En Ligne :

cours EPSSEL sur le deuil et les soins palliatifs]

Université de Franche-Comté

(2006)

\section{"Don et accompagnement professionnel dans l'action sanitaire et sociale”}

\author{
Un document produit en version numérique par Jean-Marie Tremblay, bénévole, \\ Professeur sociologie au Cégep de Chicoutimi \\ Courriel: jean-marie_tremblay@uqac.ca \\ Dans le cadre de "Les classiques des sciences sociales" \\ Site web: http://classiques.uqac.ca/ \\ Une bibliothèque fondée et dirigée par Jean-Marie Tremblay, sociologue \\ Une collection développée en collaboration avec la Bibliothèque \\ Paul-Émile-Boulet de l'Université du Québec à Chicoutimi \\ Site web: http://bibliotheque.uqac.ca/
}


Cette édition électronique a été réalisée Jean-Marie Tremblay, bénévole, professeur de soins infirmiers retraitée de l'enseignement au Cégep de Chicoutimi

Courriel: jean-marie_tremblay@uqac.ca

à partir du livre de :

Pascal Millet, “Don et accompagnement professionnel dans l'action sanitaire et sociale”. Université de Franche-Comté, cours EPSSEL sur le deuil et les soins palliatifs, 2006.

M. Millet est médecin hospitalier et professeur dans le programme : Éducation et Promotion Santé et Social en Ligne [EPSSEL, cours sur le deuil et les soins palliatifs] de l’Université de Franche-Comté.

[Autorisation formelle de l'auteur accordée le 16 septembre 2005.]

Courriel :pmillet@ch-belfort-montbeliard.rss.fr

Programme universitaire : http://epssel.univ-fcomte.fr/

Polices de caractères utilisée :

Pour le texte: Times New Roman, 14 points.

Pour les citations : Times New Roman 12 points.

Pour les notes de bas de page : Times New Roman, 12 points.

Édition électronique réalisée avec le traitement de textes Microsoft Word 2004 pour Macintosh.

Mise en page sur papier format : LETTRE (US letter), 8.5'’ x 11'’)

Édition numérique réalisée le 10 mai 2006 à Chicoutimi, Ville de Saguenay, province de Québec, Canada. 


\section{Table des matières}

Don et accompagnement professionnel dans l'action sanitaire et sociale Pascal Millet

(Cours EPSSEL sur le deuil et les Soins palliatifs)

$\underline{\text { Introduction }}$

$\underline{\text { Le don/contre-don. }}$

Le donner

Le recevoir

Le rendre (le contre-don)

La relation de don vue comme conversation

Message explicite et implicite

Les pathologies de la relation de don et contre don.

Le relation rationnelle d'échange

La relation mixte, échange et don

Don et action de soins et d'accompagnement

La relation de soins ou d'accompagnement, le don et l'identité du soignant. Les paradoxes du soin

Une expérience réelle comprend toujours une partie explicite et une partie implicite contextuelle.

Le message explicite

Le message implicite

Annexe 1. Lexique

Annexe 2. Bibliographie et lectures conseillées

Annexe 3. A rationale for the application of 'Gift-exchange' paradigm to Volunteerism by Nonprofit organisations in a Melanesian culture

Annexe 4. $\quad$ Marcel Mauss, Essai sur le don. Conclusion (Chapitre IV)

Annexe 5. Marcel Fournier, Marcel Mauss, Épinal, 10 mai 1872 - Paris, 1er février 1950 


\section{Introduction}

Marcel Mauss a initié avec «Essai sur le don » la recherche sur l'importance du don dans la société. Toutefois, à cette date, la démarche était surtout anthropologique et portait sur les manifestations du don dans des sociétés particulières (le titre complet était : Essai sur le don, forme et raison de l'échange dans les sociétés archaïques), et notamment autour de pratiques spécifiques comme le potlatch ou le kula (voir en annexe 1 -Lexique- la signification de ces mots). Ce n’est que plus récemment que des auteurs ont montré que le mécanisme du don était un mécanisme social universel et d'usage quotidien dans toutes les cultures, y compris notre culture occidentale moderne, notre culture "marchande ». (toutefois on peut consulter en Annexe 4 la conclusion de l'article de M Mauss qui montre qu'il était bien conscient de cet aspect).

Comme nous le verrons le mécanisme du don est surtout attaché à la sphère privée, familiale, en opposition avec la sphère publique, où prédominerait la relation rationnelle d'intérêt et de recherche de maximisation de cet intérêt personnel (socialité primaire versus socialité secondaire).

Or, les soignants sont souvent à l'interface de ces deux sphères, publique pour le service rendu, technique, et privée dans tout ce qui est accompagnement, relation interpersonnelle avec les patients. C'est pourquoi leur pratique est marquée par une interpénétration des mécanismes commerciaux et de ceux du " don de soi », interpénétration souvent mal analysée par les protagonistes eux-mêmes, et par-là menant à une confusion relationnelle. 
Ce texte court, trop court certainement, se propose de résumer rapidement ce que l'on sait actuellement de cette problématique. Il propose quelques pistes supplémentaires (et personnelles) tirées de ma pratique des techniques de psychothérapie dans le deuil et le soin aux toxicomanes.

À vrai dire, les idées qui seront exposées devraient être des banalités, tant le mécanisme du don est apparent dans nos sociétés. Toutefois l'expérience montre que ces idées sont encore mal connues dans le public général et même spécialisé.

Quelques exemples :

Votre mère (femme, sœur, amie...) vous offre un CD de votre chanteur préféré. Vous la remerciez. Quelques temps après, vous trouvez une reproduction d'une peinture qu'elle aime, vous lui offrez. Elle vous remercie. Dans les deux cas, il ne viendrait pas à l'esprit du destinataire du cadeau de proposer immédiatement un remboursement du prix d'achat. De même le prix de la peinture n'est pas a priori comparable à celui du CD. Simplement, comme on dit, "les petits cadeaux entretiennent l'amitié ».

Votre vendeur habituel de CD vous signale un nouveau $C D$ de votre chanteur préféré. Vous lui achetez. Comme vous êtes un bon client, pour vous "fidéliser", il vous fait une ristourne de 10\%. À quelques temps de là, vous trouvez un vendeur de CD moins cher. Vous changez sans scrupule de fournisseur habituel. Après tout votre relation avec le premier vendeur n'était que " commerciale».

Votre vendeur habituel de CD vous signale un nouveau $C D$ de votre chanteur préféré. Comme c'est aussi un fan de ce chanteur et que vous faites tous deux partie de son fan-club, il vous l'offre gratuitement. Vous le remerciez. A quelques temps de là, vous trouvez un vendeur de CD moins cher mais vous continuez à acheter chez votre premier vendeur, parce que votre relation avec lui «dépasse le commercial ». 
Ces trois histoires banales illustrent bien trois situations différentes: le don/contre-don, la relation rationnelle d'échange (commerciale, juridiquement établie etc..), la relation mixte. Examinons les l'une après l'autre.

\section{Le don/contre-don.}

Il est intuitivement évident que la relation dans le cercle familial, et plus généralement dans la sphère privée des amis, ne doit pas être menée sur un mode commercial. Quand on fait un cadeau, on efface le prix, s'il est indiqué, et quand on le reçoit, on ne parle pas de sa valeur commerciale. "À cheval donné, on ne regarde pas les dents ». En fait, chacun sait assez bien définir ce que ne doit pas être une relation amicale ou familiale (intéressée, manipulatrice, intrusive, excessive, ostentatoire...) mais la connaissance de ce qu'elle doit être est généralement moins bien partagée. Donnons en quelques aperçus.

Selon la classification de Marcel Mauss, la relation de don a trois temps : le donner, le recevoir, le rendre.

$\mathrm{Nb}$ : Nous désignerons par la suite celui qui fait le don comme le donateur et celui qui le reçoit comme bénéficiaire. On pourrait aussi utiliser donataire mais la ressemblance de donataire et donateur peut faire confusion. Nous utiliserons également récepteur quand le sens de recevoir est prédominant, comme dans la réception d'un message.

\section{Le donner}

$\underline{\text { Retour à la table des matières }}$

Comme beaucoup de proverbes et de traditions familiales l'indiquent le donner doit suivre des règles précises : 
Il doit être spontané et désintéressé. Il ne doit donc pas être fait par obligation ou dans l'attente d'un retour. Ce point est certainement celui qui a suscité le plus de controverses. Pour beaucoup d'écoles psychologiques et sociologiques, un don qui procurerait un «bénéfice », quel qu'il soit, financier, social ou narcissique, au donateur ne serait pas un don désintéressé, donc ne serait pas un « vrai » don. Pour Derrida, par exemple, le don ne doit être reconnu comme tel ni par le donateur ni par le bénéficiaire, car reconnaître un don c'est admettre une obligation morale de rendre, et cet « intérêt » annule le caractère absolu du don. Pour Bourdieu, le désintéressement est une illusio, un mensonge fait à soi-même et qui ne se perpétue que dans le non-dit. Notons toutefois que cette exigence de désintéressement traduit essentiellement une morale "protestante » (L'homme doit agir selon sa morale mais il ne doit pas se prévaloir auprès de Dieu d'un mérite tiré de sa conduite). Dans d'autres sociétés, le caractère semi-transparent de l'intérêt social du don n'est pas réellement un problème, tant qu'il relève du non-dit.

En pratique, chacun reconnaît dans la vie courante (surtout dans le milieu familial et amical) l'existence de dons reçus comme "suffisamment désintéressés » (le don s'accompagne de marques sociales évoquant la spontanéité et l'absence de désintéressement, par exemple il n'est pas ostentatoire, il n'affiche pas sa valeur, il ne parle pas d'un don antérieur dont il serait le rendu etc...). Poser la question de savoir s’il s'agit d'un désintéressement réel ou d'une illusio est le moyen le plus rapide et le plus efficace d'ôter toute sa "poésie " au don. On se gardera donc bien de la poser. Dans certains cas, toutefois, l'interrogation sur le caractère du don est incontournable et doit être affrontée. Nous traiterons ce point un peu plus loin.

Il doit être proportionné. Proportionné à la relation, à la richesse du donateur et du bénéficiaire etc.. La relation de don a longtemps été tenue en suspicion parce qu'utilisée de façon asymétrique, dans des relations de clientélisme, de charité ostentatoire ou de paternalisme social. Lorsque le don est fait de telle façon qu'il ne puisse pas être rendu (pour des raisons diverses mais le plus souvent en raison de la " pauvreté » du receveur) il ne relève plus du mécanisme du don/contre-don mais de mécanismes de don perverti (le don sans pos- 
sibilité de contre-don). Le don du don/contre-don doit être fait selon des modalités qui permettent qu'il soit accepté et rendu (même si le rendu a un caractère purement symbolique).

Il ne doit pas être valorisé de façon apparente. Non seulement le prix ne doit pas apparaître (effacement des étiquettes etc..) mais, dans nos cultures, la valeur d'usage ne doit pas se montrer. Ainsi on offrira de préférence des cadeaux apparemment inutiles (ou, du moins, décorés de telle façon que la valeur d'usage de l'objet soit dissimulée, objets de ménage " amusants » par exemple) et non des sacs de riz ou de pommes de terre. Comme l'ont remarqué de nombreux auteurs, la «bonne » définition de cette non-valorisation implique nécessairement une démarche initiale de valorisation mais l'important est qu'elle soit non-dite.

\section{Le recevoir}

$\underline{\text { Retour à la table des matières }}$

Un don, pour être « valable » doit être reçu.

Les pratiques de «bonne réception" sont influencées par la culture. Dans certaines cultures il est approprié de n’envoyer qu'un simple accusé de réception, dans d'autres il est bon de remercier longuement. Toutefois, au-delà de la diversité des cultures, les messages de base sont relativement universels :

Le don est accepté. L’utilisation des bonnes pratiques de réception atteste que le don est accepté.

Le don n'est pas accepté. L'utilisation de pratiques inappropriées (à la culture du pays) permet souvent de le signifier. Ainsi dans nos cultures occidentales, la non-acceptation du don peut être marquée par un refus exprimé ou une indifférence excessive mais aussi, au contraire, par des remerciements excessifs ou inappropriés, par exemple insistant sur la valeur matérielle du don. 
La politique internationale donne l'exemple de dons des pays riches vers les pays pauvres, qui sont vécus par ces derniers, à tort ou à raison, comme des dons humiliants (parce qu'ils ne sont pas symétriques, l'un donne et l'autre ne pourra jamais rendre). On assiste alors à des refus « inexplicables » de cette aide ou à un manque de gratitude tout aussi « inexplicable ».

Il faut noter, nous le verrons plus amplement, que les aléas de la communication peuvent distordre les messages. Ainsi l'acceptation du don (émise) peut elle être reçue comme un refus. Par exemple, une personne d'une politesse excessive pourra-t-elle donner l'impression, erronée, de se moquer du donateur. Inversement un message de refus de don, traduit par un ton ironique, pourra ne pas être perçu par le donateur, qui prendra au sérieux le compliment.

\section{Le rendre (le contre-don)}

$\underline{\text { Retour à la table des matières }}$

Dans la plupart des cultures, le cycle normal du don/contre-don suppose un rendu au don initial, un contre-don. Toutefois certaines règles s’appliquent à ce rendu :

Il est facultatif. Un rendu « obligatoire » (que ce soit par une obligation culturelle ou une législation explicite) place dans une situation relationnelle rationnelle voire commerciale et non plus de don/contredon. C'est vrai au moment du don (un don dans des circonstances de rendu obligatoire n'est pas un vrai don) ou du rendu (l'expression d'une obligation au moment du rendu « je vous le dois bien » évoque au premier chef un refus d'accepter le don).

De ce fait le rendu doit être (ou du moins apparaître comme) :

Spontané. Il ne doit même pas apparaître comme une obligation morale de rendu, pour avoir sa pleine valeur le rendu doit apparaître comme totalement spontané : «Si, si, cela me fait plaisir !! ». 
Non relié « mécaniquement » au don. Notamment un intervalle de temps adéquat (variable selon les cultures et les circonstances) doit être respecté entre le don et le rendu et le lien avec le don ne doit pas, en principe, être mentionné. Donc bien qu'il s'agisse fonctionnellement d'un rendu le contre-don doit apparaître comme un don à part entière.

Non proportionné au don. C’est une différence essentielle avec la relation commerciale, faite en principe « au juste prix ». Dans le rendu la valeur du rendu doit normalement être supérieure à celle du don, parfois inférieure et si elle est égale cela doit apparaître comme par hasard. De ce fait, le rendu peut avoir une valeur immatérielle, par exemple remerciements, manifestations de confiance etc... Le rendu peut même ne pas être proprement "rendu » par le bénéficiaire. Par exemple dans l'accompagnement des mourants ou des déments le donateur (bénévole d'accompagnement donateur de son temps et de sa présence) ressent un retour ("l'accompagnement des mourants m'a beaucoup apporté » disent ces bénévoles) , sans qu’il y ait nécessairement participation active du bénéficiaire.

Les caractéristiques attendues du rendu diffèrent selon les cultures et les circonstances, mais la société détermine habituellement sans ambiguïté si, de son point de vue, un rendu est fait correctement ou non. Mais c'est parfois à tort, c'est-à-dire en interprétant de travers les intentions de celui qui rend.

On peut s'interroger sur « l'utilité " personnelle et/ou sociale de la relation de don/contre-don. Pour de nombreux économistes, il s'agirait d'un mode archaïque d'échange de biens matériels, heureusement remplacé dans nos sociétés modernes par la relation rationnelle d'échange. De plus, pour de nombreux sociologues, notamment après Bourdieu, le don cache une utilité matérielle masquée. Ainsi la charité pratiquée par les classes supérieures envers les classes défavorisées, bien qu'apparemment désintéressée, sert à pérenniser et à justifier les relations de classe, dont profitent ces classes supérieures. 
L'apport de Mauss et des auteurs qui ont suivi est de montrer que la relation de don/contre-don a essentiellement pour but de créer et entretenir un lien de relation :

"De plus, ce qu'ils échangent ce n'est pas exclusivement des biens et des richesses, des meubles et des immeubles, des choses utiles économiquement. Ce sont avant tout des politesses, des festins, des rites, des services militaires, des femmes, des enfants, des danses, des fêtes, des foires dont le marché n'est qu'un des moments et où la circulation des richesses n'est qu'un des termes d'un contrat beaucoup plus général et beaucoup plus permanent.» Marcel Mauss : Essai sur le don, forme et raison de l'échange dans les sociétés archaïques » 1923.

La valeur attendue du don et du contre-don ne réside donc pas dans la valeur matérielle des biens échangés mais dans le lien relationnel qu'il crée, entretient et façonne entre donateur et bénéficiaire. En ce sens il s'oppose formellement à la relation commerciale, dont une qualité fondamentale est de se terminer « pour solde de tout compte ». Au contraire, l'inégalité du donné et du rendu, loin d'être une imperfection archaïque et résiduelle est le moteur de la poursuite de la relation. D’ailleurs pour interrompre la relation, le plus facile est, quand on reçoit un cadeau, d'exiger de faire immédiatement un chèque de remboursement, pour solde de tout compte : "je ne veux rien te devoir ».

La définition suivante permet d'étudier le don dans la société réelle et non dans l'imaginaire.

Qualifions de don toute prestation de bien ou de service effectuée, sans garantie de retour, en vue de créer, nourrir ou recréer le lien social entre les personnes.

(Godbout et Caillé - L’esprit du don - Boréal - 1992)

Non seulement le don/contre-don crée et entretient la relation mais encore il la façonne. Dans une famille, la manière de donner, de rendre et de recevoir façonne largement le fonctionnement familial, qui peut être ainsi marqué par la confiance, la générosité ou au contraire l'égoïsme et la suspicion. En ce sens il est objectivement vérifié que la charité unilatérale, de type paternaliste, crée, entretient 
et façonne une société inégalitaire, et peut donc être pratiquée de manière utilitariste par les classes supérieures.

Mais toute la question est de savoir s'il faut opposer au don vicié la relation rationnelle d'échange (que ce soit dans une société «capitaliste » ou « communiste ») ou la relation de don/contre-don non viciée, démocratique, équilibrée, symétrique et réciproque.

(Notons toutefois que le don «symétrique n'est pas le seul «bon » modèle de don. Dans certaines situations asymétriques par nature, par exemple entre Dieu et ses fidèles ou entre générations, il n’est pas anormal que la relation de don/contre-don vienne " confirmer" l'asymétrie de la relation.)

Le Professeur Debout, que j'avais interrogé sur les causes potentielles du taux moindre de mortalité par suicide en Grande-Bretagne, m’a répondu que le plus grand nombre de bénévoles de l'équivalent de SOS Amitié dans ce pays (les Samaritans) montrait une plus grande solidarité sociale et pouvait donc parfaitement expliquer cette moindre mortalité. Bien entendu, la réponse technique au téléphone, et son efficacité sur les personnes en menace suicidaire, est un facteur explicatif mais le plus grand facteur d'efficacité est certainement le témoignage de solidarité qui façonne, en quelque sorte, une «autre société ». Le bénéfice des bénévoles de SOS Amitié (comme d'autres associations à but humanitaire et charitable), qui n'est pas mince, est donc à travers leur engagement de promouvoir une société plus solidaire, dont ils bénéficient de façon diffuse, notamment par la diminution globale du risque suicidaire de leurs proches. Comme le dit le proverbe, "la façon de donner vaut mieux que ce que l'on donne ». 


\section{La relation de don vue comme conversation}

En fait le modèle donner, recevoir, rendre de Mauss n'est pas vraiment approprié à la description de cette relation suivie, où au don initial (souvent " oublié » ou indéfinissable dans une relation de longue date. Dans la famille le don initial est en fait le don de la vie faite aux enfants par les mères) succèdent une suite ininterrompue de dons et contre-dons. (en fait, la relation sociale habituelle inverse l'ordre de Mauss. On reçoit d'abord, la vie et les soins comme nourrissons, on rend reconnaissance et respect comme enfant et on donne assez tardivement quand on l'autonomie psychologique et matérielle le permet.)

Je propose de la voir plutôt comme une conversation, un échange de messages répondant les uns aux autres (Bourdieu a déjà exprimé l'analogie du don et du message). On peut donc utiliser la théorie de la communication, appliquée aux messages, et l'analyse conversationnelle qui permet de décrire les lois d'une «conversation » bien formée.

Selon l'analyse classique un message comprend un émetteur, un récepteur et un canal de transmission. Ici l'acte matériel de donner, comprenant l'objet donné lui-même, constitue le canal de transmission. La signification tant au niveau de l'émetteur que du récepteur est donnée par le contexte. Le même geste matériel peut revêtir des significations opposées. Ainsi si je donne un Euro à un mendiant c'est de la charité, mais si je le donne à un homme politique c'est lui signifier que je le tiens pour corrompu.

Le but de ces échanges de message est de créer ou d'entretenir la relation, de rassurer sur la nature de cette relation (souvent de façon redondante), de la corriger si le besoin s'en fait sentir etc... E Goffman a merveilleusement décrit l'échange muet de deux personnes qui manquent de se heurter dans la rue (je souris (je fais don de mon sou- 
rire), je fais des gestes d'excuse, je fais mine de m'effacer etc..) à seule fin de signifier de façon " certaine " (et la redondance contribue à cette garantie) que mes intentions ne sont en rien hostiles, et même au-delà que j'appartiens bien à la société policée qui est censée peupler cet espace public.

De la même façon les échanges de petits cadeaux au sein d'une famille ont pour but, non d'enrichir matériellement les uns et les autres, mais de rassurer sur la présence d'un « esprit de famille ». Le bénéfice du don est avant tout de pouvoir vivre dans un monde où le don a un sens.(cela rejoint un peu la notion bouddhiste du karma , voir lexique)

\section{Message explicite et implicite}

$\underline{\text { Retour à la table des matières }}$

L’École de Palo Alto distingue dans un message deux messages distincts $=$ le message explicite et le message contextuel.

Le message explicite est le message «réel, matériel : S’il s'agit d'un message textuel c'est le texte du message, s'il s'agit d'un don c'est un objet (un CD, un bouquet), si c'est un message verbal, c'est le contenu verbal du message etc..

Le message contextuel est tout ce qui est transmis en plus, « entre les lignes ", de façon non verbale etc.. Le message contextuel est rarement précis, il évoque la colère, la dérision, etc.. mais sans l'affirmer vraiment. Il relève plutôt de l'impression que de la compréhension, il est « poétique ».

"Au fur et à mesure des entretiens que je menais, j'appris sans m'en rendre compte, à avoir de plus en plus recours à des figures de rhétorique, des métaphores, des sourires etc.. d'une manière générale à utiliser de plus en plus le langage poétique ». [A Maslow l'accomplissement de soi. Eyrolles edition 2004, page 157)]. 
Par exemple, si j'offre des savonnettes parfumées à une personne qui a la réputation d'être un peu crade, il est probable que le bénéficiaire recevra bien « tout le message » mais sans pouvoir s'en offusquer ouvertement.

L'un des avantages de cette vision est que la relation rationnelle d'échange et la relation de don ne sont plus de nature opposée mais, constituent un message global où le message explicite (la description précise de l'objet mais aussi par extension les conditions générales d'échange et de valorisation de cet objet ) peut être important et prédominant dans les situations d'échange, ou s'effacer devant le message contextuel dans les relations de don. Ainsi, bien que l'acide et le sucré soient en principe opposés, un fruit peut être à la fois acide et sucré ou à la fois ni acide, ni sucré. Il sera mieux décrit par l'évaluation indépendante de l'intensité d'acidité et par celle de douceur et par la prédominance de l'une sur l'autre que par la dichotomie « acide/sucré ». Nous y reviendrons un peu plus loin, en ajoutant notamment la notion de congruence entre le message explicite et le message contextuel.

Les règles de la succession des messages, et notamment l'obligation de répondre (dans tous les sens du mot) constitue pour l'échange de messages verbaux l'analyse conversationnelle. Celle-ci étudie la ponctuation, la prise des tours de parole, l'initiation et la conclusion d'une relation de conversation. On peut dire que la littérature sur le don représente son équivalent pour les messages de don et une certaine littérature micro-économique pour les transactions rationnelles.

Les pratiques du don comme celles de la transaction rationnelle sont spécifiques à chaque culture. Comme nous l'avons déjà exprimé, la perception du caractère approprié de répondre à un don ou de mener une transaction (délai entre le don et le contre-don, valeur du contre-don, accompagnement verbal, etc..) est généralement assez bien définie chez les acteurs d'une société donnée, bien qu'elle ne fasse que rarement l'objet d'une écriture explicite (manuel de politesse par exemple). 
(Comme plusieurs chercheurs l'ont déjà mis en évidence, la conversation est très proche du don, on donne des paroles, on accepte celles que l'on reçoit et on rend selon des normes sociales assez précises.)

L'une des fonctions essentielles de cette " grammaire du don » est de permettre la stabilité du système. On l'a vu le rendu doit être en principe supérieur au donné. En l'absence de système régulateur il y a risque de dérapage, de surenchère perpétuelle, illustrée par le phénomène du potlatch, où, comme on dit dans certaines publicités « tout doit disparaître ». Dans la vie courante, la pratique quotidienne du don/contre-don ne mène pas à une surenchère, parce que des règles assez précises en modèrent la pratique.

Cette " grammaire du don » renvoie à des analyses proches, notamment celle de la politesse (par exemple R Dhoquois La politesse, vertu des apparences) ou celle du rituel (Denis Jeffrey, Éloge des rituels, Presses de l'Université Laval, 2003).

Il est impossible de développer ici tout ce que notre culture a développé contre les dérapages possibles de la relation don, contre-don, car cela prendrait plusieurs volumes, mais il faut se souvenir que ces barrières sont essentielles à une vie sociale équilibrée. Dans le domaine du soin, elles sont également indispensables pour garder « la bonne distance professionnelle » et éviter le « burn out ».

Le résumé de l'article suivant de Mr Donnadieu synthétise tous ces points (voir à http://www.afscet.asso.fr/dgcommtu.doc pour l'article complet)

La communication inter-humaine par Gérard Donnadieu

Ancien professeur à l'Université Paris 1 (Panthéon-Sorbonne)

Résumé : On doit à la théorie systémique de la communication, élaborée par l'école de Palo Alto dans les années 60/70, d'avoir rompu avec le modèle dit de l'information où seul comptait le contenu du message, pour prendre en compte les enseignements de l'anthropologie relatifs à l'économie du don et à l'échange symbolique (mis en évidence par Marcel Mauss). La nouvelle théorie met en exergue, dans tout échange humain, la primauté de la relation sur le message. Et communiquer sur la relation passe d'abord par des formes non-verbales (gestes, mimiques 
et surtout actes) que l'on désigne par le terme de communication analogique. Les formes verbales - ou communication digitale - bien adaptées à la transmission du contenu d'un message (l'information) sont relativement pauvres pour communiquer sur la relation.

Mais si l'école de Palo Alto resitue, en la minorant, l'importance de la communication digitale dans le processus global de la communication, il n'en demeure pas moins que celle-ci joue un rôle essentiel dans l'histoire de l'humanité, en particulier à partir du moment où grâce à l'invention de l'écriture vont apparaître les grandes civilisations. Peut-on alors construire une approche systémique de la communication digitale?

Les réponses proposées par les différentes sciences du langage, en particulier la linguistique, bien qu'intéressantes s'avèrent au final réductrices et insuffisantes. Elles évacuent une dimension incontournable de cette forme de communication: l'interprétation du message en référence à l'univers de symboles et de représentations qui structure l'environnement culturel des locuteurs. Ce problème de l'interprétation, ou herméneutique, qui présuppose l'existence d'un univers symbolique est au cœur de la réflexion de H.G. Gadamer et de P. Ricœur.

Contrairement à une vision purement individualiste du comportement de l'acteur, supposé producteur autonome de ses idées et qui déciderait librement selon ses intérêts (c'est le postulat de base du libéralisme économique et de la sociologie des organisations), celui-ci agit le plus souvent sous l'emprise de représentations sociales (croyances, normes, modèles, valeurs,...) plus ou moins profondément intériorisées (sous formes consciente et inconsciente) et qui lui dictent ce qu'il convient de faire dans les diverses circonstances de la vie. Agir autrement reviendrait pour l'acteur à se couper de sa société et de son groupe d'appartenance, groupe au sein duquel il puise les éléments de son identité sociale et dont dépend son existence en tant que personne.

A cet ensemble de représentations sociales, les anthropologues ont depuis longtemps donné le nom de culture, notion essentielle pour qui veut comprendre le fonctionnement d'une société. Que l'univers symbolique de la culture conditionne fortement, voire détermine de manière quasi-impérative le comportement des hommes, voilà qui peut sembler évident. Mais d'où provient alors la "consistance" d'un tel univers qui semble se tenir au-dessus des acteurs, dans une sorte de transcendance à l'image du ciel platonicien des idées ?

Pour les positivistes, tenants du réductionnisme individualiste, un tel ordre symbolique n'a qu'une apparence d'existence. Seuls existent les individus, et la culture comme la société ne sont rien d'autre que le résultat de leurs rencontres aléatoires et fugaces. Contre ce réductionnisme, nombreux sont les systémiciens à avoir élevé une protestation et proposé une réponse plus subtile, d'inspiration ago- 
antagoniste et bien dans l'esprit de la vision globale et émergentiste de la systémique.

Si en matière de dynamique relationnelle et de régulation des systèmes, la communication inter-humaine est ce qui existe au monde de plus complexe, alors la systémique est sans doute ce qui permet de la penser le moins mal possible. Prenant à la fois en compte la globalité du phénomène (par la référence à un univers symbolique qui déborde chaque individu, chaque groupe et même chaque langue particulière et renvoie indirectement à l'histoire globale de l'humanité, à la formation de ses symboles, de ses mythes fondateurs, de ses croyances) et la diversité des relations entre éléments (dualité des langages analogique et digital, modalités multiples de chacun de ces langages, caractère ago-antagoniste de l'interaction,...) l'approche systémique de la communication déborde de toutes parts la vision positiviste centrée sur l'individu, le message, l'élémentaire.

\section{Les pathologies de la relation de don et contre don.}

$\underline{\text { Retour à la table des matières }}$

Compte tenu de la grande complexité de ses mécanismes, il n’est pas étonnant que la relation de don/contre-don puisse être " pathologique ». Nous citerons quelques exemples, parmi la multitude de ceux qui peuvent être envisagés.

Il est commode classer ces anomalies en volontaires et non volontaires.

La source de la plus grande partie des « sabotages » volontaires est la peur d'entrer en relation.

Parfois il s’agit d'une prudence légitime. Une personne détentrice d'autorité (juge, policier, enseignant) se méfiera a priori des cadeaux, qui pourraient être une tentative de corruption.

Il pourra refuser explicitement (en arguant du risque judiciaire d'accepter des cadeaux) ou indirectement, par exemple en faisant immédiatement un cadeau de même valeur. 
Dans d'autres cas, le « sabotage " traduit la peur diffuse dans nos sociétés d'une relation non contrôlée avec un inconnu. Si une personne que l'on côtoie mais avec qui on n'est pas en relation intime vous fait subitement un cadeau, la première réaction est « que me veut il ?? ». Dans nos sociétés occidentales, la tendance sera de refuser le cadeau, parce qu'on est pas près à établir une relation dans ces conditions d'incertitude.

De nombreux soignants se plaignent du côté « unilatéral » de leurs relations avec leurs collègues : "je lui fais son travail quand je peux le faire, mais il ne me demande jamais si j'ai besoin d'aide ». Très souvent il ne s'agit pas d'une paresse ou d'une indifférence mais bien d'un désir de ne pas dépasser le cadre minimal de la relation entre professionnels. Si le « donateur » est conscient dès le début de ce désir de ne pas approfondir la relation, il pourra adopter une attitude plus pragmatique, par exemple :

Ne pas « tendre la main » aux collègues aussi facilement, rester sur son quant à soi,

Initier la relation avec plus de prudence par des dons moins chargés de messages contextuels (par exemple parler des enfants)

Mais aussi ne rien changer et se dire que même si les personnes que l'on aide ne vous rendent pas la pareille cela vaut aussi la peine de vivre dans un monde qui donne sa part à l'altruisme.

Les causes involontaires sont multiples, elles peuvent résulter d'une maladresse de l'émetteur, d'une anomalie du canal de transmission ou d'une mauvaise compréhension du receveur.

Les maladresses de l'émetteur peuvent être, comme on l'a dit, un don paternaliste ou ostentatoire, qui envoie un message humiliant ou ambigu au receveur, (un cadeau trop beau pour être honnête). On peut citer aussi un parent qui donnerait à un enfant fan de rap l'intégrale des symphonies de Beethoven. Message contextuel «arrête d'écouter cette sous musique? Tu devrais aimer la vraie musique. » 
Un don bien fait doit promouvoir une relation symétrique (ne pas placer le donateur au-dessus du receveur, permettre la réciprocité etc..) et ne pas véhiculer un message contextuel agressif, humiliant etc...

La mauvaise compréhension du récepteur est symétrique de la maladresse de l'émetteur. Ici l'émetteur est " conforme » aux règles, mais pour des raisons diverses, le récepteur ne comprend pas le message de façon « habituelle ». Si j'envoie un bouquet à la maîtresse de maison qui vient de me recevoir, je suis "conforme aux normes" mais si elle déteste les fleurs (ce que je ne sais pas) le message sera probablement mal reçu. C'est un phénomène courant entre personnes de culture différente. Comme le don relève le plus souvent du message contextuel, " poétique ", du non-dit, les malentendus ne sont pas facilement révélés, dépistés et corrigés, comme dans le message explicite où l'étude analytique du texte révèle plus facilement les incohérences.

Enfin le canal de transmission (l'objet donné) peut entraîner une viciation du don. Si mon cadeau arrive cassé ou moisi, même si ce n'est en rien ma faute, il est peu probable que le cadeau soit «bien reçu ».

Le « traitement » des pathologies du don fait généralement appel à des méthodes spécifiques du mécanisme, mais il existe une "panacée », un traitement universel, c'est la métacommunication : En communiquant sur la communication elle même il est possible de dépister et d'expliciter les malentendus. Par exemple le don que l'on me fait je « le sens mal », je pense que c'est un don piège. Je peux me renfermer et «faire la gueule» mais il est plus approprié de métacommuniquer : je le prends «à la rigolade » : " dis, c'est pas un piège des fois ? ». Le donateur m'explique que non, qu'il est conscient que son don peut être ambigu mais qu'il est content de pouvoir en parler, de pouvoir lever l’ambiguïté...

Voici un exemple de présentation de travail thérapeutique dans les violences familiales : 
soutien thérapeutique du système familial dans ses efforts de changement, en valorisant la confrontation mutuelle en présence des thérapeutse, par étapes successives, en s'assurant de la protection des membres vulnérables, en proposant et suscitant des alternatives aux modèles relationnels et communicationnels dysfonctionnels, en favorisant l'expression authentique des sentiments et des émotions, en exerçant la famille à la métacommunication, en catalysant les changements des expectatives multilatérales, en favorisant la redécouverte du respect mutuel, de l'estime et de la solidarité, en laissant une chance au pardon et à la réparation (élévation du seuil de l'irréparable)

$* * * * * * * * * * * * * * * * * * * * * * * * * * * * * * * * * * * * * * * * * * * * * * * * * * * * * *$ $* * * * * * * * * * * * * * * * * *$

Un sourire

Il ne coûte rien et produit beaucoup

Il enrichit celui qui le reçoit sans appauvrir celui qui le donne

Il ne dure qu'un instant mais son souvenir est parfois immortel

Un sourire c'est du repos pour l'être fatigué, du courage pour l'âme abattue, de la consolation pour le cour endeuillé

$C$ 'est un véritable antidote que la nature tient en réserve pour toutes les peines

Et si l'on refuse le sourire que vous méritez, soyez généreux, donnez le vôtre

Nul, en effet, n'a autant besoin d'un sourire que celui qui ne sait pas en donner aux autres

(le don d'un sourire. Auteur inconnu)

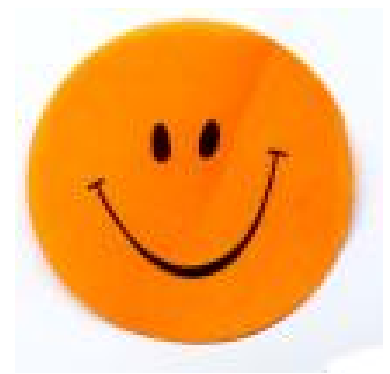




\section{La relation rationnelle d’échange}

\section{$\underline{\text { Retour à la table des matières }}$}

Chacun d'entre nous, du moins dans les sociétés occidentales, est censé être un homo economicus, un homme rationnel, faisant en permanence des choix rationnels, de façon à optimiser l'utilité de ces choix. Une large littérature " économique » vient à l'appui de cette description. Toutefois cette notion demande à être nuancée :

Aristote, à une époque ancienne, différenciait, pour un objet donné, sa valeur d'usage et sa valeur d'échange. Si la valeur d'usage peut être décrite de façon rationnelle, la valeur d'échange est largement fondée sur une convention sociale et non sur des qualités matérielles de l'objet. Ainsi certains objets, les épices, l'argent, l'or ont vu leur valeur varier dans le temps, valeur d'échange et par voie de conséquence valeur (non-dite) dans la relation de don. Ainsi, il y a quelques siècles, offrir une boîte de poivre était un cadeau somptueux, aujourd'hui cela surprendrait la maîtresse de maison. Quant à la valeur d'usage elle est toujours essentiellement de poivrer les plats.

L'utilitarisme (qui affirme la primauté de la recherche de l'utilité dans les relations sociales) a deux volets.

L'utilitarisme individuel illustré par la " main invisible » du marché d'Adam Smith. La poursuite par chacun de ses intérêts individuels contribue, de façon « invisible », à façonner l'intérêt collectif.

L'utilitarisme collectif, décrit notamment par Rawls dans sa Théorie de la Justice. Les normes "morales » permettent que l'individu, dans sa conduite, cherche à optimiser directement l'intérêt collectif et, secondairement seulement, son intérêt individuel. 
Il est évident que ces deux " utilitarismes » sont en fait très différents dans leur nature et dans leurs applications pratiques à la vie sociale.

Enfin et surtout, l'observation du comportement du consommateur montre qu'il est rarement conforme à une rationalité objective. En théorie l'homo economicus recherche une information aussi complète que possible, pèse le pour et le contre de cette information, et prend une décision qui optimise son intérêt (individuel ou collectif). Si ce mécanisme est probablement bien à l'oeuvre dans la vie des entreprises, il ne l'est souvent pas chez le consommateur. L'information qui est disponible pour le grand public est rarement objective. Il suffit de mesurer le chiffre d'affaires respectif de cette information objective (par exemple dans les revues spécialisées des associations de consommateurs) à celui de la publicité. Ensuite, même en supposant que le consommateur dispose d'une information complète, les motivations de ses choix ne sont pas toujours « objectives ». On peut le voir notamment dans le marché de l'automobile, où la plupart des caractéristiques techniques présidant au choix (vitesse maximale, aptitude à la route etc..) se situent dans un registre de performances en principe interdit par la loi. Si l'homo était vraiment economicus, la publicité n'aurait pas de sens ou ne devrait être qu'une information complète. Notre système économique vit donc largement de sa propre ambiguïté.

Malgré ces nuances, le modèle de l'homme occidental moderne est bien l'homo economicus, non seulement dans la littérature économique spécialisée, mais encore dans toute la littérature "grand public » et, pour beaucoup de personnes, le système du don/contre-don relève d'un mode relationnel archaïque, toléré dans les relations familiales mais " en voie de disparition » dans la vie sociale publique (à la grande satisfaction de ceux qui n’y voient qu'une charité paternaliste).

Pour le médecin que je suis, confronté à la réalité des relations humaines, il me semble donc que la relation rationnelle d'échange ne se distingue pas tant par son caractère calculatoire et optimalisé, que par son caractère verbalement défini et analysable. "Ce qui se conçoit bien s'énonce clairement » 
Même si la relation d'échange a un côté irrationnel et partiellement aléatoire, je peux définir précisément quels sont les éléments présidant aux choix (y compris les déterminants de l'efficacité de telle ou telle publicité), quelle est la rationalité des différents choix et en décrire la variabilité par des méthodes statistiques objectives. On peut décrire une relation d'échange (ses termes et ses déterminants), on évoque la valeur d'un don.

\section{La relation mixte, échange et don}

$\underline{\text { Retour à la table des matières }}$

Dans la réalité quotidienne, il est aisé de voir que les relations inspirées par le don restent présentes, étroitement entremêlées aux relations d'échange, même au sein de notre monde économique. Ainsi la publicité s'appuie-t-elle largement, dans ses mécanismes, sur des notions de don et contre-don. Le publicitaire fait « don » de l'esthétique, de l'humour, de la complicité et en attend un contre-don, l'acte d'achat. Le commerçant fidélise sa clientèle en signifiant à ses clients qu’ils sont « plus que des clients ». Le bénévole fait don de son temps, le politique don de sa personne et le bon vivant don de sa bonne humeur...

Voir l'article qui suit, qui montre l'interpénétration de l'échange et du don dans le temple de la Nouvelle Economie, la Silicon Valley:

CAPITAL-RISQUE ET ETHIQUE (2)

$4 / 1 / 2004$

2ème partie des interventions à la commission AFIC Venture. L'article qui suit analyse les réseaux relationnels de la silicon valley à la lumière de l'anthropologie sociale, et montre que les relations entre entrepreneurs et investisseurs ne sont pas exclusivement contractuelles. 
Les capital-risqueurs : animateurs des réseaux d'innovation de la Silicon Valley

Sur le site de Leonardo Fiances

Auteur : M Ferrary, professeur au CERAM Sophia Antipolis

\section{LA LOGIQUE D'ÉCHANGE ENTRE LES ACTEURS DES RÉSEAUX D'INNOVATION}

\section{II.1. La dynamique d'échange dans les réseaux}

L'observation de la Silicon Valley pourrait faire croire que l'opportunisme des acteurs économiques et les mécanismes concurrentiels du marché sont ceux qui régulent le management de l'innovation et la circulation de l'information. L'information est un bien qui peut s'acheter et les échanges peuvent être formalisés par des contrats. Lorsqu'un entrepreneur crée une entreprise dans la Silicon Valley, il rédige un business plan, il lève des fonds en échange d'une participation à son capital, il paye un juriste pour toutes les procédures juridiques, il loue les services d'un chasseur de tête pour recruter des salariés et il sous-traite certaines de ses activités en contractant avec des prestataires de services (comptables, relations publiques, créateur de sites web,...). En réalité, les échanges marchands d'information ne sont pas la forme d'échange la plus fréquente. Il y a de nombreux flux d'information non-monétarisés et informels entre les agents composant les réseaux socio-économiques de la Silicon Valley. Par exemple, certains juristes sont prescripteurs d'entreprises auprès des capital-risqueurs, les capitalrisqueurs sont prescripteurs pour les chasseurs de tête,... sans que pour autant il n'y ait de contrepartie financière à l'information donnée. La nature des échanges est plus complexe à la fois dans sa nature, dans son terme et dans sa dimension sociale. Le pur échange marchand n'est qu'une forme d'échange qui est complet, financier, instantané (ou à terme de paiement fixé) et inter-individuel. Il est la forme d'échange la plus simple. A l'instar de Mauss (1924) qui analysa les échanges dans des tribus amérindiennes et polynésiennes, il faut donc concevoir l'échange marchand comme n'étant qu'une dimension d'un échange plus riche dans lequel l'individu optimise certes ses ressources économiques mais également ses ressources symboliques et psychologiques. L'échange marchand ne se comprend pas en lui-même, il faut le recontextualiser dans son environnement économique, social et institutionnel. Cette socialisation amène à reconsidérer la nature et la dynamique de l'échange. Il est possible de définir quatre natures d'échange. La première est la forme pure d'échange marchand: l'acheteur et le vendeur utilisent le marché pour signaler leur désir de transaction. Pour payer le bien (produit, service ou information) acheté l'acheteur utilise un moyen financier (billet, chèque, lettre de crédit,...). L'échange est complet instantanément car les termes du contrat lève toutes les incertitudes. La seconde est lorsqu'un échange marchand est socialement déterminé: l'acheteur et le vendeur utilisent leurs réseaux sociaux pour signaler leur volonté d'échange et confronter leur offre et leur demande. Pour payer le produit acheté l'acheteur utilise également un moyen 
financier. L'échange est aussi complet instantanément. La troisième nature relève du don/contre-don de biens économiques: L'acheteur et le vendeur utilisent leurs réseaux sociaux pour signaler leur désir de transaction et confronter leur offre et leur demande. On parlera de don/contre-don de biens économiques pour deux raisons. D'une part, parce que la compensation du bien échangé n'est pas financière mais est constituée d'un autre bien économique (on est proche du modèle du troc). D'autre part, le don ne se traduit pas par une compensation immédiate mais par une compensation différée dont la nature n'est pas défini au moment de l'échange. Par exemple, un entrepreneur veut recruter un responsable commercial. Il en parle à son capital-risqueur ou à un ami, qui le met en contact avec un candidat potentiel. Si l'entrepreneur recrute le responsable commercial, il n'aura payé aucun intermédiaire pour avoir l'information. En revanche, il se sera crée implicitement une obligation à l'égard de ces personnes. Il éteindra sa dette en effectuant un contre-don (par exemple, en présentant un nouvel entrepreneur à son capital-risqueur). Pour sa part, le capital-risqueur fait le don car il anticipe que le receveur pourra faire le contre don.

La quatrième nature concerne le don/contre-don de biens non-économiques (symboliques, psychologiques,...). Les acteurs économiques sont aussi des individus avec des aspirations non-économiques relevant du besoin psychologique et/ou du besoin de reconnaissance sociale qui seront satisfaits par des échanges avec d'autres individus. Ces besoins sont bien souvent non-achetables. Dans ce cas le "bien" échangé n'est pas économique et n'appelle pas une compensation financière. Dans la Silicon Valley, on assiste à ce genre d'échanges symboliques. Tel entrepreneur invitera tel juriste dans son conseil d'administration, qui en contrepartie l'invitera sur son bateau ou à son club de golf. Tel doyen d'université fera admettre tel capital-risqueur au conseil d'université parce que ce dernier aura fait don de plusieurs millions de dollars à l'université. Concernant la dynamique des échanges, il faut les analyser dans leur répétition. Un échange marchand peut entraîner un échange-don de biens économiques, qui lui-même peut entraîner un don de biens non-économiques. Par exemple, un chasseur de tête permettra à un chef d'entreprise mélomane de le rejoindre au conseil d'administration de l'orchestre philharmonique de San Francisco et, tacitement, ce dirigeant se sentira obligé de recourir aux services de ce chasseur de tête pour une mission de recrutement dans son entreprise. Les échanges non-économiques permettent d'encastrer la relation d'échange économique dans un environnement social plus dense et modifie la nature de l'échange pour le faire évoluer d'une logique marchande à une logique du don.

Si l'on se réfère à l'approche anthropologique de Mauss, dans l'échange par le don, l'individu qui veut recevoir est obligé de donner préalablement et celui qui reçoit est obligé de rendre. Celui qui donne n'a pas de certitude sur la capacité de l'autre à rendre, ni quand et comment le don lui sera retourné. En revanche, l'agent économique qui est incapable de rendre le don, sera exclu des échanges futurs non seulement avec son partenaire mais également avec les autres mem- 
bres de la communauté que constitue le réseau. Ce qui pourrait apparaître comme un don gratuit parce qu'il ne donne pas lieu à une compensation immédiate est en fait un paiement d'un don ultérieur. Ainsi, tel capital-risqueur appellera un ingénieur d'un grand groupe industriel pour valider son analyse d'un business plan et celui-ci acceptera de lui donner ces informations sans être payé sous forme monétaire. Cette gratuité est illusoire car le don s'inscrit dans un système d'échange réciproque plus complexe et le capital-risqueur devra tôt ou tard rendre le don.

Enfin, l'échange étant inscrit dans un réseau social dense, cette forte socialisation va influer sur l'échange et sur le comportement des individus par des mécanismes de prescription et de réputation. La fonction du réseau est de réduire l'incertitude dans les échanges entre ses membres. La fréquence des interactions économiques et la densité des relations sociales permet de lever l'incertitude liée au hasard moral en permettant d'anticiper précisément les comportements individuels. La répétition des relations crée la confiance qui permet des échanges d'information qui ne seraient pas possibles dans une pure relation de marché. Par exemple, personne ne "vendra" à un investisseur l'information selon laquelle tel entrepreneur est malhonnête ou alcoolique en revanche il est possible d'acquérir l'information dans le cadre d'une relation informelle.

Dès lors qu'un individu a intégré un réseau social, il doit tenir compte de la diffusion de l'information au sein de ce réseau. Un échange avec l'un des membres d'un réseau social correspond à un échange indirect avec l'ensemble du groupe social. Dans un réseau, l'individu n'optimise pas uniquement la rentabilité de sa relation interindividuelle mais la rentabilité de sa relation avec le réseau. Certains comportements peuvent paraître économiquement irrationnels du point de vue de l'optimisation de l'échange mais deviennent parfaitement rationnels dès lors qu'on les replace dans une logique d'optimisation des échanges avec l'ensemble des membres du réseau et pas des échanges interindividuels. Dans un réseau social il est rationnel d'être honnête car l'effet réputation induit par un comportement opportuniste va exclure l'individu des échanges futurs les plus rentables. Plus l'individu sera socialisé dans le groupe social avec lequel il échange et plus le coût d'un comportement opportuniste sera élevé. Le droit contractuel sécurise l'échange marchand et non pas l'échange par le don. C'est la socialisation qui sécurise l'échange par le don par la création d'effet de réputation. Dans la Silicon Valley, de nombreux événements sociaux publics ou semi-privés contribuent à la socialisation des acteurs économiques: conférences, congrès, association, conseils d'administration,.... Ce sont des lieux institutionnalisés de socialisation où chacun vient chercher "gratuitement" des informations et où se construisent les réputations sur la capacité des membres du réseau à rendre les dons qui leur sont faits et à se conformer aux règles implicites de la communauté. Enfin, certains capital-risqueurs, travaillent toujours avec les mêmes juristes et les mêmes banques d'affaires. Les meilleurs de chaque catégorie travaillent ensemble créant des réseaux de ressources plus riches (ainsi le capital-risqueur Kleiner, 
Perkins, Caufield and Byers, le cabinet d'avocats Wilson et Sonsini, et la banque d'affaires Goldman Sachs collaborent régulièrement au service des mêmes startups). Les agents économiques se constituent en réseaux économiques pour optimiser leurs ressources individuelles et dans ce cas n'est admis dans le réseau qu'un détenteur d'une ressource qui peut être utile aux autres membres de la communauté. Par exemple, un capital-risqueur de la Silicon Valley a levé un nouveau fond d'investissement de plus de cinq milliards de francs. Les investisseurs potentiels représentant sept fois le montant recherché, le capital-risqueur à choisi parmi les investisseurs en fonction des contributions non-monétaires aux futurs investissements. Ainsi les dirigeants d'entreprises, les experts technologiques, les universités ont été privilégiés au détriment des fonds de pension ou des compagnies d'assurance.

\section{CONCLUSION:}

\section{L'ENJEU DU PREMIER DON POUR PÉNÉTRER UN RESEAU SOCIAL}

Dans la Silicon Valley, l'appartenance ou non aux réseaux d'innovation va être un facteur déterminant de l'efficience des acteurs économiques et ce quelles que soient leurs activités. Pour cette raison, tout nouvel acteur économique arrivant dans la région n'aura de cesse que de pénétrer ces réseaux pour bénéficier des informations qui y circulent et de la solidarité de ses membres. L'idéal est d'établir une relation d'échange avec un capital-risqueur car sa centralité dans les réseaux d'innovation permet de bénéficier de la richesse de son capital social. Pénétrer dans un réseau suppose, d'une part, d'amener une ressource complémentaire et, d'autre part, de respecter les règles informelles d'échange.

L'analyse anthropologique du don faite par Mauss montre l'enjeu que constitue le premier don pour établir une dynamique d'échange. Pour pénétrer les réseaux sociaux de la Silicon Valley, les nouveaux arrivants vont devoir donner aux membres des réseaux afin d'instaurer une obligation à rendre. Cependant, comme dans les tribus mélanésiennes, il est toujours possible de refuser le premier don et signifier par la même un refus d'échanger avec celui qui a fait le don. En refusant le don, le receveur évite d'avoir à le rendre et ainsi d'amorcer une dynamique d'échange. Pour que le premier don soit accepté par un capital-risqueur, il faut que ce dernier n'ait pas déjà accès à la ressource apportée. Dans ce cas, il acceptera ce premier don et s'engagera implicitement dans une dynamique d'échange. Parfois, la situation s'inverse. Les capital-risqueurs, sachant qu'ils disposent de ressources auxquels veulent accéder les autres acteurs économiques, ils vont obliger les autres à faire le premier don. Par exemple, un capital-risqueur peut contacter un chasseur de tête qu'il ne connaît pas pour avoir des informations sur un entrepreneur sans avoir à payer monétairement l'information. Le chasseur de tête sollicité acceptera de faire le premier don demandé car il sait que par la même il oblige le capital-risqueur à lui retourner le don dans le futur (invitation à siéger à un conseil d'administration, mission de recrutement,...). Le raisonnement tenu par le chasseur de tête est anticipé par le capital-risqueur et explique son 
comportement. Dans leur stratégie d'infiltration des réseaux d'innovation pour réaliser de la veille technologique, les grandes entreprises peuvent amener de nombreuses ressources spécifiques complémentaires. Elles peuvent apporter un marché nécessaire au développement d'une start-up, elles peuvent amener un accès privilégié à une technologie (par exemple, Intel donne un accès exclusif à son dernier microprocesseur six mois avant de le mettre sur le marché aux startups qu'elle finance et qui développent des logiciels qui utiliseront son microprocesseur), elle peuvent favoriser un développement à l'étranger ou racheter la start-up (en 1999, la vente de sa participation à une grande entreprise a été pour les sociétés de capital-risque une modalité de sortie des capital-risqueurs du capital des start-ups plus utilisée que l'introduction en bourse).

\section{BIBLIOGRAPHIE}

Aoki M. (1998), "Information and Governance in the Silicon Valley Model", Working Paper, Stanford University, 27 p.

Hellmann T. and Puri M. (1999), "The interaction between Product Market and Financing Strategy : The role of Venture Capital", Working paper, Stanford University, $33 \mathrm{p}$.

Mauss M. (1924), "Essai sur le don", Année Sociologique, t.1

Mais dans la mesure où, on le voit, l'intérêt est bien perceptible sous la relation, s'agit-il encore d'une relation de don, contre-don?

Pour les relations familiales et amicales (socialité primaire) il est commun d'observer des relations de don "suffisamment désintéressées ». Bien entendu une analyse rigoureuse fait apparaître, presque toujours, un "intérêt » matériel ou symbolique, et notamment l'apparition d'une obligation (de rendu, de relation, de reconnaissance etc..) mais on admet généralement que, dans ce contexte, l'absence apparente de calcul de la part du donateur suffit à rendre le don « suffisamment bon ».

Il en est autrement dans les relations publiques (socialité secondaire). De nombreux auteurs, notamment d'inspiration marxiste, ont dénoncé les fausses apparences du don public (de la charité pratiquée par les classes supérieures, du paternalisme, du prétendu don de soi..) et il est difficile à l'homme occidental un tant soit peu cultivé de les ignorer. Même quand l'intérêt matériel est exclu (comme par 
exemple dans le don aux associations d'aide aux malades, aux pauvres, au Tiers Monde...) il est aisé de soupçonner un bénéfice narcissique. Comme Narcisse, au bord de l'eau, on admire son image, ici non de beauté physique mais de générosité « désintéressée ». C’est que le don résiste mal à l'examen rationnel. Derrida voudrait que le vrai don soit « absolu », qu'il soit libéré de toute idée de restitution, de création d'obligation fut elle purement morale, d'utilité relationnelle ou narcissique,... mais alors qui peut donner en dehors d'un saint François d'Assise?

«Les temps sont durs mais modernes» dit un proverbe italien. L'individu moderne veut bien qu'on lui reproche beaucoup de choses mais certainement pas d'être naïf. Il serait même tout sauf ça. Il sait bien, lui, ce qui se cache derrière les mythes, derrière les beaux et grands récits de tous les pays et de tous les temps. L'individu moderne est réaliste. Il sait donc aussi ce qui se cache derrière le don. Ayant le triste mais moderne privilège de regarder la réalité en face et de n'être pas abusé par les faux-semblants, il sait bien que ce qui motive la production et l'échange des biens, ce n'est pas l'altruisme ou la générosité mais l'intérêt matériel » (Godbout et Caillé - L'esprit du don : Introduction).

Quelles sont les réponses données par les philosophes ou les sociologues à cette interrogation?

Nous avons vu que Bourdieu accepte le principe du désintéressement du don, mais seulement comme une illusion que l'homme se fait à lui-même, une illusio.

Cette dé-légitimation globale du processus de don lui permet certes " d'exister » » en pratique mais le rend vulnérable en cas de crise. Comment justifier la valeur intrinsèque du don, si celle ci n'est qu'illusion?

Paul Fustier, dans son ouvrage « le lien d'accompagnement », propose une solution pragmatique :

En posant brutalement l'alternative, transaction salariale (socialité secondaire) ou bénévolat (socialité primaire) le directeur détruit ce 
qui caractérisait la situation (nb référence à une situation décrite dans le texte), à savoir qu'un échange par le don venait se lover ou s'enchâsser à l'intérieur d'un système géré par le contrat salarial. Il fallait que cet élément reste implicite ou non-dit pour que l'ensemble puisse continuer à fonctionner. A l'inverse une "parole de directeur » officialisait en quelque sorte le don, et donc le détruisait en lui donnant une place reconnue dans le marché des transactions...Quand un directeur oppose brutalement temps de travail et temps bénévole, il somme souvent l'équipe éducative d'être "généreuse » et de donner du temps. Venant d'un directeur, il s'agit d'une incitation ou même d'une injonction. Dès lors l'équipe éducative est paradoxée: on ne saurait être généreux par obligation, on ne saurait donner de son temps gratuitement s'il s'agit d'un ordre... refuser c'est être avaricieux, mais accepter c'est être dans la soumission et non dans le don.

Un directeur doit pouvoir méditer l'exigence formulée par Winicott :

"Je demande qu'un paradoxe soit accepté, toléré, et qu'on admette qu'il ne soit pas résolu. On peut résoudre le paradoxe, mais le prix à payer est la perte de la valeur du paradoxe ».

P Fustier note que, dans la pratique de l'éducateur, revient souvent la question (formulée par les personnes prises en charge) " c'est pour moi que vous faites cela ou vous le faites parce que vous êtes payé pour cela ?? ». À cette question, il est donc essentiel de ne pas apporter de réponse (ou une réponse qui ne lève pas l'ambiguïté).Mais comme il le note, quelques lignes plus loin :

Ce n'est pas la "réalité » de l'échange qui fait «vérité » et caractérise le lien notamment soignant ou » éduquant ", mais l'interprétation spontanée de cet échange, telle que les protagonistes se la formulent à partir de ce qu'ils peuvent en ressentir.

Maurice Godelier, dans une perspective plus anthropologique rapporte le don à l'inaliénabilité de certains objets (l'énigme du don Flammarion - 1996). 
"Ainsi dans la pensée des Baruya, dans leurs récits sur l'origine des flûtes et les fondements du pouvoir des hommes, est présente une idée essentielle qui rattache leur conception de l'origine de l'ordre social à tout ce que nous avons dit du caractère inaliénable des objets sacrés et des objets précieux. Les premiers, nous l'avons montré, sont inaliénables et ne peuvent être donnés. Les seconds sont inaliénables et peuvent l'être. Et dans ce cas, ce qui est donné, ce n'est pas la propriété de l'objet qui reste attachée à son propriétaire d'origine mais le droit d'en user. Le donateur donne le droit d'user et garde le droit de propriété. Il garde en même temps qu’il donne » (page 182)

Nous pouvons retrouver cette idée dans les rapports modernes du don et du religieux : activité soignante des religieux etc...

Alain Caillé, dans le cadre du Mauss (Mouvement anti-utilitariste en Sciences Sociales) apporte des distinctions entre l'intérêt pour et l’intérêt à :

Je peux avoir de l'intérêt pour le jeu de poker, sans avoir intérêt à y jouer (je ne joue pas pour de l'argent). L'inverse est évidemment possible.

Je peux donc manifester un intérêt pour faire des cadeaux à mes proches (l'intérêt pour le jeu trouve une analogie dans l'intérêt que j'éprouve pour la vie dans une société solidaire et " chaleureuse »), sans que j'ai intérêt à le faire.

Alain Caillé distingue également le don de la donation. :

Voulant penser le don comme antithétique à l’intérêt égoïste calculé, posant qu'il n'est de don que là où s'évanouissent calcul et intention, la pensée moderne n'accepte de parler de don que si quelques chose qui n'existait pas apparaît sans cause, sans raison, comme coulant de source et allant de soi. Or, cet allant de soi, ou plutôt ce coulant de source, dont l'exemple principiel est la vie même (et la mort) n'est sans doute pas de l'ordre du don, mais de ce que, suivant l'usage phénoménologique (si nous ne le comprenons pas trop mal), il est possible d'appeler la donation.... La vie nous est « donnée » par nos 
géniteurs, mais elle excède infiniment ce don. Cet excès est celui de la donation sur le don...

En dernière analyse, ce qui fait la valeur d'un don, au-delà de son utilité matérielle, de sa valeur de signe, et même au-delà de sa valeur de lien, c'est le fait qu'il symbolise une dimension de donation, qu'il affirme une participation à l'univers du sans-cause, de l'inconditionné, de la vie même. L'impossible ce n'est pas le don, c'est l'identification achevée du don à la donation.

Alain Caillé - Don, intérêt et désintéressement. La Découverte /MAUSS 1994, 2005.

Citons aussi les philosophes et les religieux

Quand à l'aspect bouddhiste, après avoir médité sur les inconvénients d'un mode de vie et de pensée trop égocentrique comme sur les conséquences positives d'une attention au bien être de tous les autres êtres sensibles, une fois convaincu de l'utilité de travailler dans leur intérêt, il existe une pratique spécifique qui s'appelle " donner et porter », destinée à accroître puissance de compassion et amour envers autrui. Il s'agit d'abord de se visualiser en train de s'approprier toute la souffrance, la douleur, la négativité et les expériences indésirables das autres êtres sensibles. Dans un deuxième temps, on s'imagine que l'on porte tout cela sur ses épaules puis qu'on l'abandonne, qu'on le donne pour partager avec autrui pour ses propres qualités et ses propres inclinaisons positives: état d'esprit vertueux, énergie positive, prospérité, bonheur et ainsi de suite.... Intégrer cette pratique à son vécu quotidien est une technique très puissante dont l'influence peut avoir des conséquences extrêmement positives sur le mental et sur la santé.

Dalai Lama - La puissance de la compassion pp83-84

Il se trompe celui qui croit que donner soit chose facile. C'est une affaire qui présente beaucoup de difficultés, si toutefois on veut donner avec réflexion, et non prodiguer au hasard et par boutade. J'oblige l'un, je m'acquitte envers l'autre ; celui-là je le secours ; celui-là je le plains; cet autre je l'équipe; car il ne mérite pas d'être courbé sous la pauvreté, d'être tenu sous sa domination .Il en est à 
qui je ne donnerai pas, quoiqu'ils en aient besoin ; car, lors même que je leur aurai donné, ils auront encore besoin. Il en est à qui j'offrirai ; il en est $m^{\wedge}$ me à qui j'imposerai. Je ne puis, dans cette affaire mettre de la négligence ; jamais je ne fais de meilleur placement que quand je donne. Eh quoi, dis-tu, c'est pour recouvrer que tu donnes ? Bien mieux, c'est pour ne pas perdre.

Sénèque De la vie heureuse

Voici comment le Seigneur me donna, à moi frère François, la grâce de commencer à faire pénitence. Au temps où j'étais encore dans les péchés, la vue des lépreux m'était insupportable.

Mais le Seigneur lui-même me conduisit parmi eux ; je les soignais de tout mon cœur ; et au retour, ce qui m'avait semblé si amer s'était changé pour moi en douceur pour l'esprit et pour le corps. Ensuite j'attendis peu, et je dis adieu au monde.

Et le Seigneur me donna une grande foi aux églises, foi que j'exprimais par la formule de prière toute simple :

Nous t'adorons, Seigneur Jésus-Christ, dans toutes tes églises du monde entier, et nous te bénissons d'avoir racheté le monde par ta sainte Croix.

Ceux qui venaient à nous pour partager cette vie distribuaient aux pauvres tout ce qu'ils pouvaient avoir ; pour vêtement ils se contentaient d'une seule tunique, doublée de pièces à volonté au dedans et au dehors, plus une corde et des braies.

Et nous ne voulions rien de plus.

Nous célébrions l'office : les clercs comme les autres clercs, les laïcs en récitant le Notre Père. Et nous passions très volontiers de longs moments dans les églises.

Nous étions des gens simples, et nous nous mettions à la disposition de tout le monde.

Moi, je travaillais de mes mains, et je veux travailler ; et tous les frères, je veux fermement qu'ils s'emploient à un travail honnête.

Ceux qui ne savent pas travailler, qu'ils apprennent, non pour le cupide désir d'en recevoir salaire, mais pour le bon exemple et pour chasser l'oisiveté. 
Lorsqu'on ne nous aura pas donné le prix de notre travail, recourons à la table du Seigneur en quêtant notre nourriture de porte en porte.

Pour saluer, le Seigneur m'a révélé que nous devions dire : Que le Seigneur vous donne sa paix!

Les frères se garderont bien de recevoir, sous aucun prétexte, ni églises, ni maisons, ni tout ce qu'on pourrait construire à leurs intentions, sauf s'ils ne font qu'y séjourner comme des hôtes de passage, des pèlerins et des étrangers, conformément à la sainte pauvreté que nous avons promise dans la Règle.

Quiconque observera ces choses, qu'il soit béni dans le ciel de la bénédiction du Père très haut, qu'il soit rempli sur la terre de la bénédiction de son Fils bien-aimé, avec celle du très saint Esprit Paraclet, de toutes les Vertus des cieux et de tous les saints.

Et moi, frère François, votre petit pauvre et serviteur, dans toute la mesure dont j'en suis capable, je vous confirme, au dedans et au dehors, cette très sainte bénédiction.

Extraits du testament de saint François d'Assise.

Ces quelques éléments ne constituent qu'un échantillon des idées soulevées dans ces ouvrages. Nous renvoyons donc à la lecture complète de ces auteurs.

Nous l'avons déjà signalé, cette exigence de désintéressement est en partie culturellement déterminée et répond largement au refus protestant du mérite (très présent dans la culture anglo-saxonne). Ironiquement, un contre exemple (cf Annexe 3 pour le texte en anglais) vient de Papouasie-Nouvelle Guinée, région Pacifique ouest d'où Mauss a tiré une bonne partie de ses exemples .

L’application d'un bénévolat « intégral » (ou presque) par un organisme australien entraîne un conflit avec le personnel local, qui estime que les termes de l'échange ne sont pas équilibrés (l'organisme ne leur rend pas à la valeur de ce qu'ils y apportent). On peut penser que les australiens pensent en terme de don, opposé au salariat, alors que le personnel papou, confronté à des difficultés d'existence et d'exercice, a une vision plus globalisante, qui oppose moins nettement don et intérêt. 
Ceci illustre la tendance de nos sociétés à opposer «artificiellement » les extrêmes, le bien contre le mal, le don contre l'intérêt, le corps et l'esprit. Les cultures asiatiques ont une vision plus globalisante, illustrée par le signe du yin et du yang (voir le drapeau sud coréen). Quelle que soit la façon dont une droite coupe le cercle, elle ne se trouve jamais exclusivement dans le yin ou le yang.

De la même façon si on regarde le don comme un message comprenant une composante explicite et une (ou des) composante implicite ou contextuelle, on voit qu'il n'est pas possible de réduire le message à une seule modalité. Un cadeau qui n'aurait pas de substrat matériel (objet, parole, sourire etc..) ne serait qu'un cadeau virtuel. Mais il n'est pas possible de réaliser une transaction matérielle sans qu'un message implicite ne soit créé. Vouloir éliminer d'une transaction commerciale tout message contextuel est en soi même un message fort : "je ne veux rien avoir à faire avec vous, hors cette transaction ». Ici, le message minimaliste serait non de rien dire du tout mais d'accompagner la transaction de procédures standard de politesse, sans déroger en rien au "minimum syndical ». Comme le dit Paul Watzlawick « on ne peut pas ne pas communiquer».

On peut donc analyser toute relation d'échange, non comme relevant soit du don, soit de l'échange mais comme relevant d'une combinaison originale de divers types de messages, en combinaison ou en opposition.

Ainsi, un chirurgien (un infirmier, un avocat, un plombier etc..) dans sa pratique professionnelle peut il faire vraiment « cadeau » de sa compétence. La plus grande partie de la compétence s'acquiert par l'expérience, la rigueur, la formation etc..., toutes choses qui relèvent d'actes matériels volontaires (se former, écouter, se discipliner, donner de son temps etc..). Si l'acquisition de ces compétences ne se traduit pas directement par une modification du contrat explicite qui le lie à ses patients (fixation des honoraires notamment) on peut dire que, dans une certaine mesure, l'acquisition de ces compétences est un don fait à ses patients. Ce type de don n'est donc en rien exceptionnel. 
Il pourrait en être de même de l'acquisition de qualités relationnelles ou du respect attentif des horaires de rendez vous. (L'exactitude n'est pas une politesse que chez les rois). Elle en a le caractère spontané, non obligatoire, elle n'est pas valorisée par le donateur, elle n'appelle pas de rendu " obligatoire » (mais ne l'interdit pas), elle ne relève pas nécessairement d'un calcul d'intérêt.

Une vision idéologique classerait à coup sûr cette relation chirurgien-patient dans le cadre des relations commerciales, mais il est évident qu'il y a une place dans cette relation pour un grand nombre de messages relevant du don (aussi bien dans un sens positif que négatif. Le chirurgien peut aussi , dans les mêmes conditions de contrat, faire « cadeau » de négligence ou de laisser aller).

Ce qui relève du don est généralement tout ce qui ne relève pas du contrat ou de l'obligation sociale (explicite ou basé sur la législation ou les usages sociaux en vigueur). Il relève donc largement des messages contextuels, non verbaux et non dits. Les anciens en faisaient le domaine du sacré et du don un sacrifice, une fabrication de sacré (mais il ne faut probablement pas commettre l'erreur d'attribuer au mot «sacré » la signification spécialisée qu'il a acquis dans notre monde moderne. Je propose que nous définissions ici comme sacré ce qui ne relève pas du « matériel »).

Sacrifice, étymologiquement « fait de rendre sacré » (du latin sacrificium, de sacer facere).

(Si nos sociétés occidentales ne sacrifient plus de bœufs, ses membres continuent à faire le sacrifice de leur temps, de leur week end et parfois même de prix sacrifiés).

Un des points importants d'analyse de ces relations est la congruence des messages entre eux. Congruence entre le message explicite et le ou les messages contextuels mais aussi entre les messages contextuels entre eux.

Dale Carnegie dans son ouvrage culte «Comment se faire des amis et Réussir en affaires » disait que pour reussir en affaires, il fallait vouloir le bien du client : 
(Ce qui fait la différence entre un commercial et un commercial d'élite, c'est la capacité de ce dernier à instaurer un climat de confiance basé sur une relation privilégiée (publicité du Dale Carnegie Training Center)) mais il ajoutait que ce qui est important c'est de le penser vraiment.

Le caractère analogique du message contextuel lui fait emprunter de nombreux canaux. Ainsi le caractère bienveillant d'un don pourra $\mathrm{t}$ il être évoqué par l’objet donné, par les paroles, le ton de la voix mais dénié par l'attitude corporelle qui évoque l’ironie.

Le caractère congruent des messages associés au don est donc une caractéristique importante, notamment dans le soin et l'accompagnement. Toutefois il ne garantit pas la « sincérité » du don puisque cette congruence peut être obtenu par des moyens techniques. La congruence est une qualité essentielle de l'acteur de théâtre ou de cinéma mais aussi de l'escroc, du menteur ou du tricheur.

Toutefois une caractéristique essentielle de la congruence est qu'elle est essentiellement perçue, donc qualifie ce qui est reçu plus que ce qui est émis.. La congruence permet donc au bénéficiaire de maintenir l'illusion (dans le langage de Bourdieu mais appliqué au bénéficiaire et non plus au donateur) d'un don désintéressé. On le voit notamment dans les grands hôtels où la politesse formelle du personnel est largement techniquement apprise (et relève donc du contrat salarial) mais où sa congruence permet de maintenir l'ambiguïté du don « spontané » de la disponibilité et de la volonté de servir.

Toujours dans le langage de Bourdieu, et de façon certainement trop schématique, le désintéressement est l'illusio du donateur et la congruence l'illusio du bénéficiaire. D'une certaine manière, la congruence, (dans le langage de Paul Fustier le mécanisme principal du maintien de l'ambiguïté, de la non résolution du paradoxe) est un don supplémentaire du donateur au bénéficiaire. Remarquons que sincérité et congruence entretiennent une relation fonctionnelle habituelle mais non exclusive. On peut être perçu comme congruent sans être sincère, par technique acquise, et comme non congruent, par maladresse, malgré une grande sincérité. 
Même au niveau du donateur, on pourrait définir le don « véritable " comme congruent à la déclaration de spontanéité et d'absence d'intéressement. Toutefois, chacun connaît des professionnels qui, derrière un discours cynique et apparemment totalement " commercial », déploient des trésors de générosité. L’exemple inverse est malheureusement fréquent de donateurs " généreux » dont les motivations se révèlent rapidement frauduleuses.

Au delà du modèle du don et de l'échange, l'analyse conversationnelle, étendue aux relations matérielles et non seulement verbales, mais surtout ce qui concerne le don, constitue le fondement même de l'identité de nos sociétés et des individus qui la composent.(voir notamment le compte rendu de lecture de l'ouvrage de J Godbout)

Le don, la dette et l'identité

\section{Homo donator vs. Homo æeconomicus Jacques T. GODBOUT,}

Dans L'Esprit du don, Jacques T. Godbout montrait que le don occupe encore une place de première importance dans nos sociétés, à côté du marché et de l'État. Dans ce nouvel ouvrage, il généralise son propos : le don est ce mode de circulation des biens et services propre aux réseaux et où n'intervient pas la séparation entre un public et des professionnels. Dans la famille ou dans la société, le monde des réseaux fonctionne au don et à la dette, et non pas à l'équivalence (comme dans le marché) ou à l'égalité (comme dans l'État). Quand les réseaux fonctionnent bien, cette dette est positive : elle n'engendre pas angoisse et aliénation, mais confiance et désir de loyauté. Le don apparaît ainsi indissociable du sens : c'est l'intention qui compte et c'est le sens qui fait le don. Enfin, c'est à travers la relation de dette (positive ou négative), de don et de contre-don, que se forment ou se déforment les identités.

Nourri par de nombreuses recherches empiriques, ce livre propose ainsi un véritable paradigme alternatif à celui de la science économique et de la sociologie utilitariste. Au lieu de postuler que nous serions tous des homo æeconomicus, qui ne songent qu'à prendre et à garder, il risque l'hypothèse inverse : ne serions-nous pas plutôt du genre homo donator, davantage motivés en fait à donner qu'à recevoir ?

Sans aucune pose théoriciste, modestement et avec rigueur, Le don, la dette et l'identité prend à contre-pied un grand nombre d'idées reçues dans les sciences sociales. 
Jacques T. GODBOUT, professeur-chercheur à l'Institut national de la recherche scientifique (Université du Québec), est l'auteur de La participation contre la démocratie (Saint-Martin), La démocratie des usagers (Boréal) et, en collaboration avec Alain Caillé, de L'Esprit du don (La Découverte/Boréal). Considéré comme un des meilleurs spécialistes mondiaux de la sociologie du don, J. T. Godbout est membre du comité de rédaction de La Revue MAUSS.

La conversation impose le respect de règles précises, dans une société donnée, tour de parole, ne pas interrompre une séquence, proportionner la réponse à la demande initiale etc.., ce qui assure qu'un dialogue n'est pas la superposition de deux monologues. Le terme " dialogue de sourds » illustre bien ce que devient une conversation sans règles (et non sans oreilles).

De la même façon les règles de l'échange de dons permettent une harmonie de l'échange, éloignant aussi bien l'absence de réponse (qui met fin à la relation) que la réponse excessive, qui pourrait mener rapidement à un cercle vicieux. De ce fait, la relation de don n'est pas exempte de toute évaluation (l'évaluation des dons et contre-dons étant nécessaire à l'harmonisation) mais cette évaluation est au service de la relation plus que de l'échange (elle cherche à optimiser la relation pas la valeur de l'échange matériel). Dans la relation de soins le déséquilibre des termes de l’ échange est un des déterminants du « burn out » des soignants.

\section{Don et action de soins et d'accompagnement}

Retour à la table des matières

Je l'ai déjà dit ailleurs, le travail de soin est au système de santé ce que le travail domestique est à l'économie marchande ; essentiel mais invisible, omniprésent mais sans valeur marchande. D'ailleurs la valeur marchande qu'on lui attribuerait ne serait peut-être pas équitable. Car, justement, l'identité de soignante c'est celle de l'identité à l'Autre. Les soins sont avant tout une pratique relationnelle, qui ne se déploie que dans une relation, une relation sociale qui transporte avec elle des formes de lien créatrices d'identité entre deux individus ou des groupes d'individus, et c'est dans cette rencontre qu'une part de l'invisibilité sociale se construit. .. La relation de soin crée par sa structure un tiers (le plus que l'un et l'autre, c'est à dire l'un, l'autre et 
le relation porteuse de lien) et, sans ce tiers, elle n'opère pas. Elle ne peut qu'être invisible, difficilement saisissable, parce que fugitive, marquée par le temps de la rencontre. Sans commencement ni fin, parce qu'inscrite dans la circularité de ce qui doit vivre et peut être, être donné. Et cette question du don et de la circulation du don dans les soins est complexe, difficile à appréhender, et se doit d'être approfondie. Particulièrement quand le don n'est pas d'ordre matériel, mais renvoie au temps, à la vie elle-même ou au sacré »

Francine Saillant et Eric Gagnon - Responsabilité pour autrui et dépendance dans la modernité avancée. Le cas de l'aide aux proches. Lien social et politiques $\mathrm{N}^{\circ} 46$, 2001

Et, pourtant, même dans la sphère marchande, le soin représente une part non négligeable.

Le français moyen "acquiert » tous les ans une valeur supérieure à 300 euros en salaires de soignants, sans même compter le recours au bénévolat associatif ou familial.

Nous illustrerons cette problématique par le rapport entre le don et l'identité du soignant. Nous tenterons ensuite de proposer une solution aux paradoxes du soin.

\section{La relation de soins ou d'accompagnement, le don et l'identité du soignant.}

$\underline{\text { Retour à la table des matières }}$

(Les soignants professionnels se distinguent en principe des bénévoles ou des accompagnants familiaux. Toutefois, en pratique, le travail de bénévole demande une compétence technique, ce qui les rapproche des professionnels. De plus, pour de nombreux sociologues, Bourdieu ou Derrida notamment, leur engagement procède d'un « intérêt caché ", sociologique, personnel voire narcissique. C'est pourquoi, dans cette analyse, nous ne distinguerons pas les professionnels des bénévoles, sauf mention explicite. 
Malgré une forte protocolisation des actions de soins et d'accompagnement dans nos sociétés modernes, chaque personne soignée peut témoigner que chaque acte de soins et d'accompagnement (en comprenant l'accompagnement familial et bénévole) comporte un caractère qui lui est propre, une identité. Car la prestation technique n'est qu'une partie d'une expérience globale. Certes, entre le soin bref et technique dans une maladie bénigne et de courte durée d'une part et l'accompagnement dans une maladie grave ou mortelle d'autre part, le vécu de la relation de soins n'est pas comparable mais il n'en reste pas moins que chaque rencontre est une expérience unique, irréductible à toute description exhaustive.

À travers sa pratique quotidienne, et donc l'exercice de relations multiples et répétées, le soignant ou le bénévole va donc se créer une identité personnelle de soignant, une façon d'être habituelle, un habitus pour utiliser un terme cher aux sociologues.

Mais quelle identité ? On peut distinguer la personne du rôle. L'identité du soignant n'est pas nécessairement celle de la personne (qui intègre tous les rôles, familiaux, sociaux, professionnels etc..) : "Quand il est chez lui, on ne le reconnaît pas » dira un collègue de travail. On peut aussi distinguer le rôle du personnage. Pour certains chercheurs le don sincère relève du rôle et le don calculé du personnage, qui serait donc ce que le tricheur fait de son rôle. Toutefois cette analyse, bien que séduisante parce qu'elle permettrait en principe de " décider » de la sincérité de la relation, ne me paraît pas applicable en pratique.

Le rôle est en partie ce que le sujet (ici le soignant) décide de faire, mais aussi ce que la société lui impose à travers les normes morales ou professionnelles, normes qu'il incorpore dans son habitus. Il y a donc une distance entre le rôle théorique, idéal, et la pratique quotidienne du soignant. On retrouve la distinction que fait l'ergonomie entre la tâche prescrite et la tâche effectuée. Il y a souvent une distance, voire parfois un gouffre, entre ce que le travailleur est censé faire et ce qu'il fait réellement. L'étude de cette différence, loin d'être un facteur de confusion, est un moyen puissant d'analyser et d'optimiser le service rendu. On peut donc assimiler le rôle à la tâche prescrite 
et le personnage à la tâche telle qu'elle est effectuée en pratique. La différence entre les deux n'est pas toujours liée à une « tricherie » volontaire, elle peut aussi bien être liée aux insuffisances du soignant ou au caractère trop idéal du rôle défini, à l'insuffisance de moyens ou de soutien de l'environnement etc... La distance du rôle au personnage nous semble donc un phénomène " obligé », dont l'analyse extérieure ne permet pas d'affirmer le caractère volontaire. Seul le soignant luimême peut savoir ce qu'il en est, s'il dispose de l'honnêteté intellectuelle que cela requiert. Nous distinguerons donc chez le soignant la personne, le rôle et le personnage comme trois « identités » différentes au moins (il peut y avoir des rôles et des personnages multiples selon les circonstances).

$N b=$ les termes de rôle, personne et personnage peuvent être utilisés différemment (avec d'autres significations) par certains chercheurs

Selon la théorie de la communication, sur laquelle nous reviendrons, les messages (comprenant le don) que l'une de ces identité envoie à la personne soignée sont aussi envoyées à ces autres identités. Ainsi les rebuffades (don négatif) que le personnage soignant inflige au soigné pourront elles être vécues négativement par la personne du soignant (mais vues comme ne relevant pas de la personne s'ils sont en rapport avec un manque de moyens dans la pratique des soins) et bien sûr comme contraires à son rôle idéal de soignant.

Les relations difficiles entre ces trois entités engendreront une " souffrance » du soignant et c'est souvent ce type de causalité qui est mis en évidence par l'analyse de la pratique soignante :

"Ce qui me gêne le plus ce n'est pas le salaire ni les heures passées, c'est que je ne peux matériellement pas donner les soins que je devrais faire, et surtout je ne peux pas donner le temps nécessaire à écouter mes malades, à leur parler. Je ne suis qu'une technicienne des soins.»

Inversement, certains soignants vivent une «bonne » harmonie entre les aspects techniques, contractuels et organisationnels de leur pra- 
tique d'une part et la qualité de leur vécu relationnel d'autre part. Ce sont des soignants « heureux ».

La relation unique dans chaque pratique de soins entre ce qui relève du geste technique, médical, et du contrat de soins, et de tout ce que le soignant apporte "en plus » par un don de temps, d'attention, de bienveillance, de bonne humeur, de disponibilité... et le patient par un don de reconnaissance, de tolérance et de "participation aux soins ", tout cela définit, de façon unique et irréductible à toute description exhaustive, une situation de soins. Si l'on définit l'invisibilité comme l'absence de reconnaissance verbale et explicite (voire d'évaluation), alors, en effet cette relation de soins comprend beaucoup d'invisible. Mais pour ceux, soignants et soignés, qui vivent cette relation de soins, il s'agit réellement d'une "expérience totale ». Nous renverrons donc chacun, soignant ou soigné, à la singularité de son vécu personnel. Ce " plus » de la relation soignante est assez proche du sentiment artistique, comme le montre le texte suivant :

Au travail, la reconnaissance est fondée sur deux jugements :

- Le premier est un jugement d'utilité. On reconnaît que ce que je fais est utile. Ce jugement est porté par le client, l'élève, le malade, et par la hiérarchie. Il n'est absolument pas évident que tous les salariés bénéficient de ce niveau de reconnaissance. Mais ça n'est pas tout. L'identité ne se règle pas exclusivement dans la dimension de l'utilité.

- Un deuxième type de jugement intervient. Le jugement esthétique : "ce travail est un travail bien fait !", "c'est du beau travail !". Ce jugement est le jugement des pairs. Ainsi, chaque fois que vous êtes confrontés à une situation inhabituelle, difficile, qui impose une mobilisation particulière, si vous y arrivez, vous n'avez rien de plus pressé que de trouver quelqu'un qui soit en position de mesurer ce qu'il y avait de difficile et de juger de ce que vous avez fait, afin qu'il valide votre action, qu'il la reconnaisse (Davezies, 1992).

Le jugement esthétique comporte lui même deux dimensions (Dejours, 1993a).

- C'est un beau travail parce que c'est un travail conforme aux règles qui constituent le collectif de travail. Cette dimension sanctionne l'appartenance à la communauté, premier versant de l'identité.

- Mais aussi, c'est un beau travail parce qu'il comporte quelque chose de singulier. Parce qu'il y a un apport personnel, parce que ce n'est pas la stricte re- 
production de ce qu'on fait habituellement. C'est le deuxième versant de l'identité. L'identité en tant que je ne suis justement identique à aucun autre.

\section{Éléments de psychodynamique du travail -Philippe Davezies}

Laboratoire de médecine du travail. Faculté de médecine, rue G. Paradin, 69732 Lyon cedex 08

Texte diffusé à la réunion de la Société de médecine du Travail de MidiPyrénées

Toulouse 13 avril 2000

(Inspiré de Dejours, notamment Dejours C - Le Facteur Humain PUF 1995 ou DEJOURS C. (1993) : Pour une clinique de la médiation entre psychanalyse et politique: la psychodynamique du travail. Communication à la Société psychanalytique de Montréal. Revue TRANS. 3 : 131-156).

\section{Les paradoxes du soin}

$\underline{\text { Retour à la table des matières }}$

La relation de soins suscite, nous l'avons vu, de nombreuses interrogations: relève-t-elle du contrat professionnel ou du don de soi, relève-t-elle d'une aptitude technique acquise ou " vient elle du coeur ", doit elle entraîner un contre-don de la part du soigné, et si oui lequel, ou au contraire est ce,seulement la satisfaction narcissique du « devoir accompli » qui est recherchée par le soignant ???

Nous essaierons de répondre en utilisant le modèle de l'expérience totale. 


\section{Une expérience réelle comprend toujours une partie explicite et une partie implicite contextuelle.}

\section{Le message explicite}

La situation de soins comprend nécessairement une relation matérielle, soins, traitements, dialogue ou même simplement écoute, qui requiert temps, présence et disponibilité. Il est indiscutable que la médecine scientifique a apporté à l'humanité une valeur ajoutée à l'acte de soins, qui a permis de doubler l'espérance de vie dans les pays développés et d'apporter l'espoir de guérison dans de nombreuses maladies, là où le désespoir était jadis la règle. Ce serait pratiquer une lecture idéologique et abusive de l'Histoire que de prétendre que toutes ces découvertes ont été motivées par l'intérêt économique des acteurs. Aujourd'hui, dans notre monde en principe motivé entièrement par l'intérêt économique, la large disponibilité sur Internet de l'information médicale gratuite montre que la plupart des chercheurs, et des institutions notamment universitaires, qui les emploient, travaillent dans un modèle de "don » du progrès à la collectivité et de gratuité. On a pu constater la mobilisation contre les essais de brevets sur ce qui est perçu comme la propriété collective de l'humanité, le code génétique ou les traitements contre le SIDA notamment. À cet égard, malgré les décisions parfois contraires de la justice et des législations, le devoir de résistance apparaît pour beaucoup comme un devoir d'humanité. C'est assez attester de la persistance de l'idée du don même dans le saint des saints de la modernité.

Plutôt que d'intérêt ou de valeur économique, je préfère donc parler de langage explicite. La partie explicite du message est ce qui peut être décrit par des mots.

Il existe à ce propos un malentendu chez beaucoup de soignants. La pratique des soins est encadrée de plus en plus par des procédures 
d'évaluation et de valorisation. De plus, pour des raisons de financement ou de notation, la tentation est grande de créer des indices synthétiques qui puissent " résumer » l'évaluation des différentes dimensions de l'acte de soins. On a ainsi créé en son temps des points ISA (Indice synthétique d'activité) qui permettaient, sur une échelle de 100 à 20000 environ, de comparer la valeur médicale du traitement de l'appendicite, de l'alcoolisme chronique, de la dépression ou de l'eczéma. Depuis cette échelle a été remplacée par une échelle en euros !

Pourquoi ne pas créer sur ce modèle un indice synthétique de compassion ?? En fait, il en existe plusieurs (voir lectures conseillées évaluation du soin). Certains sont utilisés pour évaluer l'aptitude relationnelle des élèves infirmier(e)s, d'autres pour relier les symptômes post opératoires à l'attitude empathique de l'infirmière, d'autres encore pour relier cette attitude empathique à la qualité des soins, à la satisfaction du patient ou à la satisfaction professionnelle du soignant.

Bien entendu, il n'est pas question de critiquer ces approches, qui permettent toutes d'améliorer la qualité des soins, mais de remarquer que ce qui doit être évité ce n'est pas la construction d'un index mais son usage en dehors du contexte de sa construction.

Le formulaire permet à un enseignant dévaluer la conformité à un modèle des aptitudes relationnelles et à l'élève infirmier (infirmière) de situer sa pratique relationnelle par rapport à ce modèle, il permet d'évaluer l'effet somatique de la compassion ou l'effet de la relation de soins sur la satisfaction professionnelle des soignants etc.... mais sa validité ne doit pas être étendue à d'autres circonstances, par exemple mesurer la compassion "réelle » d'un professionnel de soins ou adapter le financement d'un hospice à la "réalité » de sa prestation compassionnelle. Deux attitudes opposées naissent de la même erreur.

L'attitude "technocratique" consisterait à utiliser, sans esprit critique, un index de compassion pour classer des «bons » ou des «mauvais » hôpitaux. Malheureusement il existe chez les dirigeants, en général, une tendance à cette dérive technocratique, qui doit être surveillée de près. 
L'attitude « anti-technocratique » consiste à fantasmer sur des «significations " attribuées abusivement aux index. On accuse souvent les points ISA de ne pas "tenir compte » de la qualité réelle de la prestation de soins. Ils ne sont pas faits pour cela, ils sont faits pour évaluer les besoins de financement des hôpitaux et ils sont probablement plus efficaces et plus justes dans cette fonction que la répartition aléatoire des financements qu'ils ont remplacé. Dit on de la déclaration d'impôt qu'elle ne mesure pas la situation financière réelle des ménages ?? C'est pourtant vrai en partie mais est il utile de mesurer la situation financière réelle des ménages tant que la Loi de Finances n’est pas modifiée pour en tenir compte ??

Il ne faut donc pas opposer la prestation technique, médicale, relevant de la relation verbale, commerciale, à la relation d'empathie seule porteuse des valeurs du don. La compétence, la rigueur sont des dons, au même titre que l'empathie ou la compassion.

Quel scientifique ne rêve $t$ il pas de donner (dans tous les sens du terme) la guérison aux sidéens et pourtant ce don ne pourra se faire que dans le fonctionnement hyper-rationnel et coûteux d'un laboratoire de virologie.

Le texte suivant résume bien cette ambiguïté (reference site ssrfr.org)

Les résultats des enquêtes réalisées pour cette session le montrent bien : malgré sa spécificité, la santé suit les évolutions des représentations sociales dominantes : recherche de l'immédiateté, de l'efficacité, attente forte vis-à-vis de la science, montée de l'individualisme, levée des interdits avec des points de résistance fragiles.

L'homme moderne est avant tout pressé: le système de santé doit le prendre en charge - il y a droit - et le guérir sans délai. Même si l'amélioration de la médecine néonatale, l'allongement de la durée de la vie et une meilleure prévention conduisent inévitablement à une certaine augmentations des maladies chroniques, voire du handicap, il demande paradoxalement à " naître et mourir en bonne santé ", c'est-à-dire indépendant, autonome et libre de ses choix. La maladie qui le rend vulnérable doit être éliminée au plus vite. La vie en effet doit se mener "au top " du début à la fin. La représentation idéale de la mort est désormais celle de la mort subite, rapide, quasi indolore. La souffrance n'est plus tolérée. Son éradication est un impératif. Mieux : un devoir. Et si le discours vis-à-vis de l'accueil 
des handicapés reste politiquement correct, l'IMG apparaît comme moralement supérieur à l'IVG. L'IVG est en effet perçu comme une défaillance de la vigilance, alors que l'IMG est un moyen de "remédier aux erreurs de la nature ".

Pour répondre à ces demandes contradictoires de prise en charge globale et de réparation technicienne, le système de santé se répartit le travail : Il y a tout d'abord le généraliste, dont la fonction est de réconforter (rôle psychologique) et d'aiguiller (vers le spécialiste). Son prestige décroît en fonction de son rôle d'écoute, de compassion et de conseil. Mal rémunéré, il prescrit trop, voit trop de patients, tout ceci au risque de " passer à côté du bon diagnostic ". Vient ensuite, à l'opposé, le spécialiste, cher, compétent, (la médecine technicienne, c'est lui) mais glaçant. Enfin, dans le milieu hospitalier, une place toute particulière est faite au personnel soignant. Supposé " connaître les gestes qui réconfortent ", il n'est jamais coupable : c'est " le système ", mal géré, bureaucratique, qui est responsable. Le malade prend désormais peu à peu sa place dans cet ensemble, mais de façon ambiguë : à la fois comme partenaire, mais aussi comme client d'un service : il a des droits et le fait sentir. L'accès au dossier médical, quoi que l'on puisse penser par ailleurs de cette disposition, apparaît comme une des illustrations de cette place nouvelle du patient et de son ambiguïté.

Jean-Pierre Rosa, Mars 2002

\section{Le message implicite}

Si le message explicite est ce qui décrit par les mots, le message implicite est il ce qui ne peut pas être décrit par les mots ???

Les fleurs à inventer les jouets d'une comète

Les raisons d'être fou la folie dans ta tête

Des avions en allés vers tes désirs perdus

Et moi comme un radar à leurs ailes pendu

Des embruns dans tes yeux et la mer dans ton ventre

Un orgue dans ta voix chaque fois que je rentre

Des chagrins en couleur riant à ton chevet

Les lampes de mes yeux pour mieux les éclairer

Les parfums de la nuit quand ils montent d'Espagne

Les accessoires du dimanche sous ton pagne

Les larmes de la joie quand elle est à genoux

Le rire du soleil quand le soleil s'en fout

Les souvenirs de ceux qui n'ont plus de mémoire

L'avenir en pilules toi et moi pour y croire

Des passeports pour t'en aller t'Einsteiniser 
Vers cet univers glauque où meurent nos idées

Des automates te parlant de mes problèmes Et cette clef à remonter qui dit " je t'aime " Un jardin dans ton cœur avec un jardinier Qui va chez mon fleuriste et t'invite à dîner Des comptes indécis chez ton marchand de rêves Un sablier à ton poignet des murs qui lèvent Des chagrins brodés main pour t'enchaîner à moi Des armes surréelles pour me tuer cent fois

Cette chose qu'on pense être du feu de Dieu Cette mer qui remonte au pied de ton vacarme Ces portes de l'enfer devant quoi tu désarmes Ces serments de la nuit qui peuplent nos aveux Et cette joie qui fout le camp de ton collant Ces silences perdus au bout d'une parole Et ces ailes cassées chaque fois qu'on s'envole Ce temps qui ne tient plus qu'à trois... deux... un... zéro.

\section{JE TE DONNE TOUT ÇA, MARIE !}

Je te donne - Léo Ferré

Comme le montre par exemple l'analyse de la poésie il est toujours possible d'ajouter des mots aux mots. On peut commenter l'œuvre d'un poète, rappeler en quoi sa vie personnelle a inspiré son œuvre, analyser son emploi des mots et des tournures de phrase etc.... Mais ces mots ne remplaceront jamais l'émotion que suscite la lecture du poème, mais ils peuvent parfois «briser le charme ». Il en est de même pour tous les arts, peinture, musique... Il en est de même pour la relation de soins.

Or notre culture occidentale nous amène souvent à croire que la valeur ne réside que dans ce qui peut être décrit par les mots. Comme dit le matérialiste "voilà comme sont vraiment les choses, tout le reste c'est de la poésie ...». Revendiquons donc le droit à la poésie.

Ces moments d'intense communication que nous avons avec ce qui nous entoure - qu'il s'agisse d'une rangée d'arbres, d'une salle ensoleillée - sont en eux-mêmes insaisissables. Nous n'en jouissons que dans la mesure où nous communiquons, où nous sommes perdus, inattentifs. Si nous cessons d'être perdus, si notre attention se concentre, 
nous cessons pour autant de communiquer. Nous cherchons à comprendre, à capter le plaisir : il nous échappe. (Georges Bataille, L'Expérience intérieure.)

Comme le montre l'exemple de Derrida et de Bourdieu, entre autres, l'analyse « scientifique » des déterminants du don est le moyen le plus rapide et le plus efficace d'en détruire la " poésie ». En matière de vivant la méthode de déconstruction-reconstruction prônée par Derrida n'a jamais produit un être vivant mais, au mieux, un cadavre recousu.

Inversement, comme le dit JC Kaufmann dans l'analyse de la répartition des tâches ménagères dans le couple :

Plus généralement, les tentatives d'évaluation égalitaire entretiennent un lien des plus subtils et des plus paradoxaux avec le don. Car, outre les difficultés purement techniques de la mesure, l'essentiel est que la norme n'est jamais la même pour les deux conjoints : chacun a ses manières particulières, ses définitions du propre et du rangé. L'acceptation du mode général d'organisation du travail et de vagues normes communes de calcul représente donc toujours une concession, un don de l'un. Les comptes sont ensuite tout à fait secondaires, ils ont surtout pour but de renforcer l'impression d'égalité. Le plus important s'est déroulé avant, dans la mise au point des règles du jeu. C'est pourquoi certains couples à la volonté égalitaire affichée donnent singulièrement l'occasion d'observer des formes très prononcées de don, l'idéologie égalitaire constituant le meilleur paravent, pour que le don se structure dans le silence et la non-représentation.

JC Kaufmann, La trame conjugale. Nathan, 1992, page 166

L’irruption des mots dans cette relation conjugale est illustrée par le moment où, dans une procédure de divorce, la relation conjugale de don, même dégradée, est remplacée par l'estimation rationnelle, et même juridique, de la dette de l'un envers l'autre. Il est fréquent d'observer la dégradation immédiate de la relation entraînée par ce simple changement de perspective.

Peut être l'invisibilité de la relation de soins, mise en évidence par F Saillant, est elle due partiellement à l'inaptitude des mots à la dé- 
crire. Souhaitons donc que des mots puissent être mis sur ces dons inestimables qui relevaient jusque là du non dit. Mais souhaitons aussi que ces mots ne «tuent » pas la poésie du don de soi. La leçon de cette étude n'est elle pas que dans chaque relation de soins, aussi banale soit elle, se joue quelque chose qui relève du sacré ??

\section{Kôan 24 du Wumenguan}

Un bonze demanda à Fengxue :

«La parole et le silence sont tous les deux le fonctionnement interne et externe de l'esprit, comment s'en sortir sans se tromper? »

Fengxue répondit :

" Je me rappelle toujours la lune sur le fleuve Jiang, les perdrix y chantaient, les dix mille fleurs embaumaient l'air »

T Jyoji - L’art du Kôan zen Albin Michel 2001 


\section{Annexe 1 - Lexique}

$\underline{\text { Retour à la table des matières }}$

Accompagnement : Présence physique, psychologique et professionnelle d'un intervenant dans une phase d'adaptation, de réadaptation ou d'intégration sociale dans le but d'assurer le bon déroulement de cette phase.

Compassion Compatir, c'est "souffrir avec". La compassion n'est pas une émotion. Il s'agirait plutôt d'une attitude qui nous porte à être sensible à la souffrance de quelqu'un. Devant la douleur d'autrui, nous vivons alors différentes émotions. Il peut s'agir de tristesse, de colère, de révolte, etc..., toutes suscitées par ce que nous observons chez la personne qui pâtit. Il faut également distinguer la compassion de l'empathie. L'empathie est une attitude qui rend capable de saisir ce qu'une personne vit émotionnellement, tel qu'elle le vit. C'est en quelque sorte la capacité de se mettre à sa place pour la comprendre "de l'intérieur".

Don (nom masculin) Action d'accorder gratuitement à quelqu'un la propriété, $\underline{\text { la }}$ jouissance de quelque chose..$^{\bullet} \underline{\text { La }}$ chose ainsi accordée..$^{\bullet}$ Talent, aptitude. ${ }^{\bullet}$ Offrande. $\cdot$

Karma

Tout acte est le mûrissement d'une cause antérieure et celui-ci aura une conséquence dans le futur. Ce que nous sommes aujourd'hui est le résultat des actes passés et ce que nous effectuons maintenant, déterminera l'avenir. Cette loi de causalité du karma est ce qui conditionne toute notre existence. Cependant, tous ces conditionnements ne sont pas définitifs car nous avons une certaine part de liberté et, quelles que soient les circonstances, il demeure toujours une possibilité de choix. En résumé, il y en a deux : soit de progresser vers la lumière de l'éveil, soit de régresser vers les ténèbres de l'ignorance. C'est la raison pour laquelle nous avons le libre arbitre. Bien que nous soyons prédisposés depuis la naissance, ces propensions ne nous déterminent pas totalement. Il est donc en notre pouvoir de nous libérer ou de nous aliéner. 
Tout acte en bien ou en mal, du corps, de la parole et de l'esprit aura une conséquence bonne ou mauvaise, dans cette vie, dans la suivante ou bien après.

Il existe un très grand nombre d'actes nuisibles, mais on peut les ramener à dix. Trois pour le corps : le meurtre (et le suicide), le vol et l'inconduite sexuelle ; quatre pour la parole : le mensonge, la calomnie, les paroles blessantes et les paroles inutiles ; et trois pour l'esprit : la convoitise, la malveillance et les vues fausses sur la nature essentielle de la réalité. Les dix actes positifs consistent à abandonner les dix actes négatifs. Il s'agit de protéger la vie des êtres animés, de pratiquer la générosité, et d'avoir une conduite éthique, de dire la vérité, de créer l'harmonie entre les gens, de parler de manière pacifique et de tenir des propos sensés ; d'avoir peu de désirs tout en étant satisfait de ce que l'on a, de développer la bienveillance en soi et d'adhérer à ce qui est authentique (loi du karma, réincarnation, etc.).

En bref, il est dit dans le Dharma : "Sont négatifs les actes engendrés Par le désir, la haine et l'ignorance ; Ils sont la source de toute souffrance et des états d'existence inférieurs. Sont positifs les actes qui résultent de l'absence de désir, de haine et d'ignorance ; Ils engendrent les mondes heureux Et le bonheur dans toutes les vies. » Évidemment, une personne récoltera inéluctablement le fruit de ses actes et ne pourra pas les transférer à quelqu'un d'autre ; ceux qui sont positifs engendreront le bonheur et ceux qui sont négatifs, la souffrance. Si on peut comprendre cette notion de karma au niveau individuel, il est quelquefois difficile d'admettre que l'on ait à subir les conséquences de certains actes au niveau collectif comme, par exemple : les guerres, les fléaux, les calamités, etc. En fait, le karma collectif est la réunion de multiples karma individuels. On peut le remarquer au niveau des groupes humains qui se réunissent pour des raisons sociales, religieuses, politiques et autres ; plus il y a de gens concernés, plus l'impact est puissant.

Dans la perspective du bouddhisme, il n'y a pas de jugement ultime autre que la loi de rétribution karmique. Le destin n'est pas non plus une fatalité, mais la loi naturelle de cause à effet. Ainsi, à chaque instant de notre vie, nous pouvons transformer notre karma négatif en positif et améliorer encore plus notre karma bénéfique.

Kula . Le circuit kula des îles Trobriand, que B. Malinowski a étudié pour la première fois, est une ronde de don, car il y a des mouvements de deux articles qui circulent sans cesse dans des directions opposées (le " soulava ", sorte de longs colliers de coquillages rouges, et le « mwali ", des bracelets de coquillage blanc).

Potlatch : distribution ostentatoire de biens d'un groupe à un autre au cours d'une fête ; ce don est un défi : il implique pour celui qui le reçoit l'obligation de donner plus tard à son tour l'équivalent sous peine de perdre la face et de descen- 
dre dans la hiérarchie de prestige. Des pratiques analogues ont été observées par les ethnographes dans beaucoup de sociétés à travers le monde : c'est une forme d'échange liée à une rivalité pour le prestige. Un vestige dans nos sociétés est une obligation de "rendre une invitation". 


\title{
Annexe 2
}

\author{
Bibliographie \\ et lectures conseillées
}

$\underline{\text { Retour à la table des matières }}$

\section{Psychologie générale}

Goffman E- Les cadres de l'expérience - Les éditions de minuit 1991

Goffman E - La mise en scène de la vie quotidienne 1973

Marc E et Picard D - L'école de Palo Alto - Retz 2002

Maslow A - L'accomplissement de soi - Eyrolles 2004

Rogers C - Le developpement de la personne - Dunod 1998

Watzlawick P - Le langage du changement

\section{Le don}

Anspach $\mathrm{R}$ - a charge de revanche, figures élémentaires de la réciprocité - Le Seuil 2002

Beaucage $\mathrm{P}$ - Présentation, échange et société : avant et après Mauss dans « Retour sur le don » Revue anthropologie et sociétés Vol 19-1-2, 1995

Caillé A - Don, interet et désinterressement - La Découverte MAUSS 2005

Debra A - donner: de l'avoir à l'être ou avoir à être http://www.cefem.be/index.php/layout/print/content/view/full/271

Godbout J et Caillé A - L’esprit du don - 1992

Godelier M - L’énigme du don Flammarion 1996 
Mauss M - Essai sur le don, forme et raison de l'échange dans les sociétés archaïques - sociologie et anthropologie PUF 19985c1950, p 145,279

Saillant F - Identité, invisibilité sociale, altérité - Revue Anthropologie et Sociétés 24-1 2000 : P 155-171

\section{Applications de la théorie du don (sauf soins)}

Dhoquois R - La politesse, vertu des apparences - Autrement 1991

Kaufmann JC- La Trame Conjugale - Nathan 1992

Lardellier - Théorie du lien rituel - L'harmattan - 2003

Picard D - Politesse, savoir vivre et relations sociales PUF 1998

\section{Applications de la théorie du don (soins)}

Baum M - La relation médecin-patient : entre proximité et distance Louvain Med, 117 : S203-S209, 1998

Benbassat J, Baumal R -What is empathy and how can it be promoted during clinical clerkship ? Acad Med 2004; 79(9): 832-839

Carrut A - Development and testing of the caregiver reciprocity scale - Nursing research 45(2) : 92-97, March/April 1996

Fields et al - Comparison of nurses and physicians on an operational measure of empathy - Evaluation and the health professions vol27 $\mathrm{N}^{\circ} 1,80-94$ (2004)

Fogarty L et al - Can 40 seconds of compassion reduce patient anxiety ? - J of Clin Oncology - vol 17 Issue 1 January 1999 : 371

Frederiksson L - Modes of relating in a caring conversation: a research synthesis on presence, touc and listening J Adv Nurs 1999 Nov;30(5): 1167-76

Fustier P- Les corridors du quotidien - PUL 1993

Fustier P - Le lien d'accompagnement - Dunod 2000

Gagnon E et Saillant $F$ - De la dépendance et de l'accompagnement : soins à domicile et liens sociaux - L'Harmattan 2000

Hojat et al - Physician empathy : definition , components, measurement and relationship to gender and specialty - Am J of Psychiatry - 159:1563-1569, Sept 2002 
Kim et al - The effects of physician empathy on patient satisfaction and compliance - Evaluation and the health professions - vol27, $\mathrm{N}^{\circ} 3,237-251$ (2004)

Mercer SW et al - Empathy and quality of care - British Journal of General Practice 200252 S9-S12

Mercer SW et al - Relevance and practical use of the Consultation and Relational Empathy (CARE) Measure in general practice - Family practice 200522 328-334

Saillant $\mathrm{F}$ et Gagnon E - Responsabilité pour autrui et dépendance dans la modernité avancée. Le cas de l'aide aux proches - RIAC Automne 2001

Tschui M- Le don d'organes - Anne Carrière 2003 


\section{Annexe 3}

A rationale for the application of 'Gift-exchange' paradigm to Volunteerism by Nonprofit organisations in a Melanesian culture

Murray E Millar

7 October 2004

A rationale for the application of 'gift-exchange' paradigm to volunteerism by Nonprofit Organisations in a Melanesian culture.

Abstract

A number of Australian non-profit organisations (NPOs) operate in both Australia and in developing countries and rely upon local volunteers in each situation. It is important for these organisations to know how volunteerism by local people in developing countries compares to volunteerism within Australia so that valid assumptions underlie the work in different cultural contexts. While a considerable amount of research has been done on formal volunteering within Australia, to date there have been no comparable studies conducted on formal volunteering in Papua New Guinea (PNG). Formal volunteering is a relatively new concept for PNG people even though informal volunteering embodied in "caring and sharing", is recognised as the "Melanesian way" (Kidu 2000). This paper reports a pilot case study of an Australian NPO working in PNG that uses local PNG volunteers in a number of its programs. The results indicate that understanding of formal volunteering in PNG may be significantly different to that in Australia. PNG people who work as unpaid volunteers are regarded by community members in mixed ways. They may be perceived by some as extremely dedicated, but by others as humiliating both themselves and their communities. To be called a volunteer can even be considered insulting. 
A review of the literature relating to Melanesian culture suggests that the gift exchange paradigm (Gregory 1982; Carrier 1991) may be a fundamental influence on attitudes towards volunteerism by PNG people. This paper proposes a gift-exchange model for assisting an NPO to make formal volunteering culturally appropriate in PNG.

\author{
Murray E Millar \\ 26 Sunray Cres, Horsley, NSW 2530 AUSTRALIA \\ Home phone: (61 2) 42625972 \\ Mobile: (61) 0402726065 \\ mem37@uow.edu.au
}

\title{
Conclusion
}

This pilot case study has provided a strong indication that people's understanding and acceptance of formal volunteerism is influenced by culture. This has important implications for the way NPOs manage volunteer programs cross culturally. In PNG, while people may choose to volunteer for an organisation and express altruistic reasons for doing so, self-motivated reasons are often present as well. This is being influenced by both basic survival needs and cultural factors.

The majority of volunteers interviewed in this pilot case study were located in urban or peri-urban3 areas of PNG. A large proportion stated that they faced considerable financial challenges to meet basic needs. It is extremely difficult for people to work for communities without remuneration when they are struggling to meet their personal and family needs for food, housing, transport and education.

Unemployment is high in PNG, especially in the urban areas, with many people lacking the education and experience to enable them to gain employment. Consequently, they are often willing to work in voluntary, unremunerated positions with NPOs with the hope that this will be a means of helping them improve their employability. Evidence gained from this case study indicates that it will be rare to find people willing to volunteer for an NPO in PNG, especially in urban areas, who do not have significant unmet personal needs.

3 Peri-urban refers to locations in the edges of urban centres or in villages close to urban centres. Consequently there would be expectation of remuneration in some form or another for work undertaken.

In respect to culture, both the review of literature and the responses of informants in this pilot case study indicate that, as in $\mathrm{M}$ ori culture, reciprocity is a deeply rooted part of Melanesian culture. Through gift-exchange, relationships 
are made and maintained. It is by giving that status is achieved as the person who receives the gift is placed in a debt relationship with the giver. However, there are clear obligations to reciprocate. In some situations this is soon after the gift is received while in others it will take place over time. Within this cultural paradigm, there is little place for giving without expecting anything in return. Consequently, working voluntarily for an organisation that is not part of this giftexchange system is of no benefit to the one giving time and labour. Work that does not bring immediate return (through wages) or the possibility of return over time (as gift-exchange normally provides) is not considered honourable and contributes nothing to the status of the giver (volunteer) in the eyes of the community.

This pilot study suggests that for NPOs to work successfully with volunteers within a Melanesian cultural context, it will be important that the local practices of gift exchange be understood and taken into account. The importance of this extends beyond mere economic considerations to include social and political factors. Its significance to PNG people may be intimately linked to the basic needs for survival as described above.

To help formal volunteerism to be more acceptable and effective within a Melanesian culture, this study proposes a gift-exchange model. The volunteer and the NPO are seen as the two exchange partners. Both bring separate 'gifts' into the exchange relationship in the form of three matched pairs.

First, the NPO provides a valid, community identified cause to which a volunteer can in reciprocation freely commit.

Secondly, the volunteer comes with personal needs and goals which have been made explicit in the recruitment interview process. In reciprocation, the NPO seeks ways to flexibly meet those needs within the restraints of the broader situation while meeting the work requirements of the voluntary assignment.

Finally, the volunteer brings capabilities suitable to the work at hand and the NPO reciprocates by further enhancement of those capabilities while providing the essential resources to carry out the work with associated credible recognition.

Equality between the partners in the relationship is not considered necessary, as the volunteer and the organisation have clearly different roles and responsibilities. While the relationship that is entered into will be enduring, it does not imply that the volunteer will remain a volunteer for ever, or that the volunteer will necessarily change from volunteer status to full time working status with the organisation. However, through the relationship, the volunteer will make significant contributions

and in turn will be benefited extensively. As a result the volunteer will remain indebted to the organisation and the organisation to the volunteer. Each will freely value the gift that the other brings to the relationship. Inappropriate dependency need not develop, as in the process of exchange, the volunteers' potential as indi- 
viduals will be enhanced, and their ability to contribute to the community will be recognised. Further research into the relationship between gift-exchange and the four core elements of volunteerism could investigate questions such as:

1. What part does free choice play, in respect to volunteering, in a context where gift-exchange obligations exist?

2. For people whose social relationships are dependent on gift-exchange, what would be the effect of working voluntarily to meet the needs of people whom

one has no prior relationship with?

3. How can NPOs that are operating within gift-exchange based cultures effectively motivate people who are working for them without being paid when, as an organisation they appear like a for-profit business and clearly manage significant budgets?

4. If the voluntary principle is truly "an integral part of democratic society" (Oppenheimer 2000: 10), what would be the best way to promote volunteerism in PNG so that current stigmas are overcome and people who volunteer are highly respected and valued by community members?

This study is limited in that only people already working as volunteers were interviewed and these only within one NPO. To add validity to these conclusions it would be necessary to extend the population to encompass other NPOs, as well as people who are not working as volunteers, and to also cover a wider geographical area, including other countries in Melanesia. It may also be helpful to test this model, using an action based research approach, and make appropriate adjustments that will enable wider application and increased effectiveness for NPOs working with volunteers in Melanesian societies.

A rationale for the application of 'gift-exchange' paradigm to volunteerism by Nonprofit Organisations in a Melanesian culture by M E Millar 


\title{
Annexe 4 \\ Conclusion de l'essai sur le don (M Mauss)
}

\author{
Don gracieux du site Les Classiques des sciences sociales. \\ (qu'ils en soient remerciés)
}

\author{
I \\ CONCLUSIONS DE MORALE
}

$\underline{\text { Retour à la table des matières }}$

Il est possible d'étendre ces observations à nos propres sociétés.

Une partie considérable de notre morale et de notre vie elle-même stationne toujours dans cette même atmosphère du don, de l'obligation et de la liberté mêlés. Heureusement, tout n'est pas encore classé exclusivement en termes d'achat et de vente. Les choses ont encore une valeur de sentiment en plus de leur valeur vénale, si tant est qu'il y ait des valeurs qui soient seulement de ce genre. Nous n'avons pas qu'une morale de marchands. Il nous reste des gens et des classes qui ont encore les mœurs d'autrefois et nous nous y plions presque tous, au moins à certaines époques de l'année ou à certaines occasions.

Le don non rendu rend encore inférieur celui qui l'a accepté, surtout quand il est reçu sans esprit de retour. Ce n'est pas sortir du domaine germanique que de rappeler le curieux essai d'Emerson, On 
Gifts and Presents ${ }^{1}$. La charité est encore blessante pour celui qui l'accepte ${ }^{2}$, et tout l'effort de notre morale tend à supprimer le patronage inconscient et injurieux du riche « aumônier ».

L'invitation doit être rendue, tout comme la «politesse ». On voit ici, sur le fait, la trace du vieux fond traditionnel, celle des vieux potlatch nobles, et aussi on voit affleurer ces motifs fondamentaux de l'activité humaine : l'émulation entre les individus du même sexe ${ }^{3}$, cet « impérialisme foncier » des hommes; fond social d'une part, fond animal et psychologique de l'autre, voilà ce qui apparaît. Dans cette vie à part qu'est notre vie sociale, nous-mêmes, nous ne pouvons « rester en reste », comme on dit encore chez nous. Il faut rendre plus qu'on a reçu. La «tournée » est toujours plus chère et plus grande. Ainsi telle famille villageoise de notre enfance, en Lorraine, qui se restreignait à la vie la plus modeste en temps courant, se ruinait pour ses hôtes, à l'occasion de fêtes patronales, de mariage, de communion ou d'enterrement. Il faut être "grand seigneur » dans ces occasions. On peut même dire qu'une partie de notre peuple se conduit ainsi constamment et dépense sans compter quand il s'agit de ses hôtes, de ses fêtes, de ses « étrennes ».

L'invitation doit être faite et elle doit être acceptée. Nous avons encore cet usage, même dans nos corporations libérales. Il y a cinquante ans à peine, peut-être encore récemment, dans certaines parties d'Allemagne et de France, tout le village prenait part au festin du mariage ; l'abstention de quelqu'un était bien mauvais signe, présage et preuve d'envie, de «sort». En France, dans de nombreux endroits, tout le monde prend part encore à la cérémonie. En Provence, lors de la naissance d'un enfant, chacun apporte encore son oeuf et d'autres cadeaux symboliques.

Les choses vendues ont encore une âme, elles sont encore suivies par leur ancien propriétaire et elles le suivent. Dans une vallée des Vosges, à Cornimont, l'usage suivant était encore courant il n'y a pas longtemps et dure peut-être encore dans certaines familles : pour que

Essais, 2e série, V.

2 Cf. Koran, Sourate II, 265; cf. KOHLER in Jewish Encyclopaedia, I, p. 465.

3 William JAMES, Principles Of Psychology, II, p. 409. 
les animaux achetés oublient leur ancien maître et ne soient pas tentés de retourner " chez eux », on faisait une croix sur le linteau de la porte de l'étable, on gardait le licol du vendeur, et on leur donnait du sel à la main. À Raon-aux-Bois, on leur donnait une tartine de beurre que l'on avait fait tourner trois fois autour de la crémaillère et on la leur présentait de la main droite. Il s'agit, il est vrai, du gros bétail, qui fait partie de la famille, l'étable faisant partie de la maison. Mais nombre d'autres usages français marquent qu'il faut détacher la chose vendue du vendeur, par exemple : frapper sur la chose vendue, fouetter le mouton qu'on vend, etc. 4

Même on peut dire que toute une partie du droit, droit des industriels et des commerçants, est, en ce temps, en conflit avec la morale. Les préjugés économiques du peuple, ceux des producteurs, proviennent de leur volonté ferme de suivre la chose qu'ils ont produite et de la sensation aiguë que leur travail est revendu sans qu'ils prennent part au profit.

De nos jours, les vieux principes réagissent contre les rigueurs, les abstractions et les inhumanités de nos codes. À ce point de vue, on peut le dire, toute une partie de notre droit en gestation et certains usages, les plus récents, consistent à revenir en arrière. Et cette réaction contre l'insensibilité romaine et saxonne de notre régime est parfaitement saine et forte. Quelques nouveaux principes de droit et d'usage peuvent être interprétés ainsi.

Il a fallu longtemps pour reconnaître la propriété artistique, littéraire et scientifique, au-delà de l'acte brutal de la vente du manuscrit, de la première machine ou de l'œuvre d'art originale. Les sociétés n'ont, en effet, pas très grand intérêt à reconnaître aux héritiers d'un auteur ou d'un inventeur, ce bienfaiteur humain, plus que certains droits sur les choses créées par l'ayant droit ; on proclame volontiers

4 KRUYT, Koopen, etc., cite des faits de ce genre aux Célèbes, p. 12 de l'extrait. Cf. De Toradja's... Tijd. v. Kon. Batav. Gen., LXIII, 2; p. 299, rite de l'introduction du buffle dans l'étable; p. 296, rituel de l'achat du chien qu'on achète membre à membre, partie du corps après partie du corps, et dans la nourriture duquel on crache ; p. 281, le chat ne se vend sous aucun prétexte, mais se prête, etc. 
qu'elles sont le produit de l'esprit collectif aussi bien que de l'esprit individuel ; tout le monde désire qu'elles tombent au plus vite dans le domaine publie ou dans la circulation générale des richesses. Cependant le scandale de la plus-value des peintures, sculptures et objets d'art, du vivant des artistes et de leurs héritiers immédiats, a inspiré une loi française de septembre 1923, qui donne à l'artiste et à ses ayant droit un droit de suite, sur ces plus-values successives dans les ventes successives de ses œuvres ${ }^{5}$,

Toute notre législation d'assurance sociale, ce socialisme d'État déjà réalisé, s'inspire du principe suivant : le travailleur a donné sa vie et son labeur à la collectivité d'une part, à ses patrons d'autre part, et, s'il doit collaborer à l'œuvre d'assurance, ceux qui ont bénéficié de ses services ne sont pas quittes envers lui avec le paiement du salaire, et l'État lui-même, représentant la communauté, lui doit, avec ses patrons et avec son concours à lui, une certaine sécurité dans la vie, contre le chômage, contre la maladie, contre la vieillesse, la mort.

Même des usages récents et ingénieux, par exemple les caisses d'assistance familiale que nos industriels français ont librement et vigoureusement développées en faveur des ouvriers chargés de famille, répondent spontanément à ce besoin de s'attacher les individus euxmêmes, de tenir compte de leurs charges et des degrés d'intérêt matériel et moral que ces charges représentent ${ }^{6}$. Des associations analogues fonctionnent en Allemagne, en Belgique avec autant de succès. En Grande-Bretagne en ce temps de terrible et long chômage touchant

5 Cette loi n'est pas inspirée du principe de l'illégitimité des bénéfices faits par les détenteurs successifs. Elle est peu appliquée.

La législation soviétique sur la propriété littéraire et ses variations sont bien curieuses à étudier de ce même point de vue : d'abord, on a tout nationalisé; puis on s'est aperçu qu'on ne lésait ainsi que l'artiste vivant et qu'on ne créait pas ainsi de suffisantes ressources pour le monopole national d'édition. On a donc rétabli les droits d'auteurs, même pour les classiques les plus anciens, ceux du domaine public, ceux d'avant les médiocres lois, qui. en Russie, protégeaient les écrivains.Maintenant, on le dit, les Soviets ont adopté une loi d'un genre moderne. En réalité, comme notre morale, en ces matières, les Soviets hésitent et ne savent guère pour quel droit, opter, droit de la personne ou droit sur les choses.

6 M. Pirou a déjà fait des remarques de ce genre. 
des millions d'ouvriers - se dessine tout un mouvement en faveur d'assurances contre le chômage qui seraient obligatoires et organisées par corporations. Les villes et l'État sont las de supporter ces immenses dépenses, ces paiements aux sans travail, dont la cause provient du fait des industries seules et des conditions générales du marché. Aussi des économistes distingués, des capitaines d'industries (Mr. Pybus, sir Lynden Macassey), agissent-ils pour que les entreprises elles-mêmes organisent ces caisses de chômage par corporations, fassent ellesmêmes ces sacrifices. Ils voudraient en somme, que le coût de la sécurité ouvrière, de la défense contre le manque de travail, fasse partie des frais généraux de chaque industrie en particulier.

Toute cette morale et cette législation correspondent à notre avis, non pas à un trouble, mais à un retour au droit ${ }^{7}$. D'une part, on voit poindre et entrer dans les faits la morale professionnelle et le droit corporatif. Ces caisses de compensation, ces sociétés mutuelles, que les groupes industriels forment en faveur de telle ou telle oeuvre corporative, ne sont entachées d'aucun vice, aux yeux d'une morale pure, sauf en ce point, leur gestion est purement patronale. De plus, ce sont des groupes qui agissent : l'État, les communes, les établissements publics d'assistance, les caisses de retraites, d'épargne, des sociétés mutuelles, le patronat, les salariés ; ils sont associés tous ensemble, par exemple dans la législation sociale d'Allemagne, d'Alsace-Lorraine ; et demain dans l'assurance sociale française, ils le seront également. Nous revenons donc à une morale de groupes.

D'autre part, ce sont des individus dont l'État et ses sous-groupes veulent prendre soin. La société veut retrouver la cellule sociale. Elle recherche, elle entoure l'individu, dans un curieux état d'esprit, où se mélangent le sentiment des droits qu'il a et d'autres sentiments plus

7 Il va sans dire que nous préconisons ici aucune destruction. Les principes de droit qui président au marché, à l'achat et à la vente, qui sont la condition indispensable de la formation du capital, doivent et peuvent subsister à côté des principes nouveaux et des principes plus anciens.

Cependant il ne faut pas que le moraliste et le législateur se laissent arrêter par de soi-disant principes de droit naturel. Par exemple il ne faut considérer la distinction entre le droit réel et le droit personnel que comme une abstraction, un extrait théorique de certains de nos droits. Il faut la laisser subsister, mais la cantonner dans son coin. 
purs : de charité, de "service social ", de solidarité, Les thèmes du don, de la liberté et de l'obligation dans le don, celui de la libéralité et celui de l'intérêt qu'on a à donner, reviennent chez nous, comme reparaît un motif dominant trop longtemps oublié.

Mais il ne suffit pas de constater le fait, il faut en déduire une pratique, un précepte de morale. Il ne suffit pas de dire que le droit est en voie de se débarrasser de quelques abstractions : distinction du droit réel et du droit personnel ; - qu'il est en voie d'ajouter d'autres droits au droit brutal de la vente et du paiement des services. Il faut dire que cette révolution est bonne.

D'abord, nous revenons, et il faut revenir, à des mœurs de « dépense noble ». Il faut que, comme en pays anglo-saxon, comme en tant d'autres sociétés contemporaines, sauvages et hautement civilisées, les riches reviennent - librement et aussi forcément - à se considérer comme des sortes de trésoriers de leurs concitoyens. Les civilisations antiques - dont sortent les nôtres -avaient, les unes le jubilé, les autres les liturgies, chorégies et triérarchies, les syssities (repas en commun), les dépenses obligatoires de l'édile et des personnages consulaires. On devra remonter à des lois de ce genre. Ensuite il faut plus de souci de l'individu, de sa vie, de sa santé, de son éducation chose rentable d'ailleurs - de sa famille et de l'avenir de celle-ci. Il faut plus de bonne toi, de sensibilité, de générosité dans les contrats de louage de services, de location d'immeubles, de vente de denrées nécessaires. Et il faudra bien qu'on trouve le moyen de limiter les fruits de la spéculation et de l'usure.

Cependant, il faut que l'individu travaille. Il faut qu'il soit forcé de compter sur soi plutôt que sur les autres. D'un autre côté, il faut qu'il défende ses intérêts, personnellement et en groupe. L'excès de générosité et le communisme lui seraient aussi nuisibles et seraient aussi nuisibles à la société que l'égoïsme de nos contemporains et l'individualisme de nos lois. Dans le Mahabharata, un génie malfaisant des bois explique à un brahmane qui donnait trop et mal à propos : "Voilà pourquoi tu es maigre et pâle. » La vie du moine et celle de Shylock doivent être également évitées. Cette morale nouvelle consistera sûrement dans un bon et moyen mélange de réalité et d'idéal. 
Ainsi, on peut et on doit revenir à de l'archaïque, à des éléments ; on retrouvera des motifs de vie et d'action que connaissent encore des sociétés et des classes nombreuses : la joie à donner en publie ; le plaisir de la dépense artistique généreuse ; celui de l'hospitalité et de la fête privée et publique. L'assurance sociale, la sollicitude de la mutualité, de la coopération, celle du groupe professionnel, de toutes ces personnes morales que le droit anglais décore du nom de «Friendly Societies » valent mieux que la simple sécurité personnelle que garantissait le noble à son tenancier, mieux que la vie chiche que donne le salaire journalier assigné par le patronat, et même mieux que l'épargne capitaliste - qui n'est fondée que sur un crédit changeant.

Il est même possible de concevoir ce que serait une société où régneraient de pareils principes. Dans les professions libérales de nos grandes nations fonctionnent déjà à quelque degré une morale et une économie de ce genre. L'honneur, le désintéressement, la solidarité corporative n'y sont pas un vain mot, ni ne sont contraires aux nécessités du travail. Humanisons de même les autres groupes professionnels et perfectionnons encore ceux-là. Ce sera un grand progrès fait, que Durkheim a souvent préconisé.

Ce faisant, on reviendra, selon nous, au fondement constant du droit, au principe même de la vie sociale normale. Il ne faut pas souhaiter que le citoyen soit, ni trop bon et trop subjectif, ni trop insensible et trop réaliste. Il faut qu'il ait un sens aigu de lui-même mais aussi des autres, de la réalité sociale (y a-t-il même, en ces choses de morale, une autre réalité ?) Il faut qu'il agisse en tenant compte de lui, des sous-groupes, et de la société. Cette morale est éternelle; elle est commune aux sociétés les plus évoluées, à celles du proche futur, et aux sociétés les moins élevées que nous puissions imaginer. Nous touchons le roc. Nous ne parlons même plus en termes de droit, nous parlons d'hommes et de groupes d'hommes parce que ce sont eux, c'est la société, ce sont des sentiments d'hommes en esprit, en chair et en os, qui agissent de tout temps et ont agi partout.

Démontrons cela. Le système que nous proposons d'appeler le système des prestations totales, de clan à clan, - celui dans lequel individus et groupes échangent tout entre eux - constitue le plus ancien système d'économie et de droit que nous puissions constater et concevoir. 
Il forme le fond sur lequel s'est détachée la morale du don-échange. Or, il est exactement, toute proportion gardée, du même type que celui vers lequel nous voudrions voir nos sociétés se diriger. Pour faire comprendre ces lointaines phases du droit, voici deux exemples empruntés à des sociétés extrêmement diverses.

Dans un corroboree (danse dramatique publique) de Pine Mountain ${ }^{8}$ (Centre oriental du Queensland), chaque individu à son tour entre dans le lieu consacré, tenant dans sa main son propulseur de lance, l'autre main restant derrière son dos ; il lance son arme dans un cercle à l'autre bout du terrain de danse, nommant en même temps à haute voix le lieu dont il vient, par exemple : «Kunyan est ma contrée » ${ }^{9}$; il s'arrête un moment et pendant ce temps-là ses amis "mettent un présent », une lance, un boomerang, une autre arme, dans son autre main. « Un bon guerrier peut ainsi recevoir plus que sa main ne peut tenir, surtout s'il a des filles à marier ${ }^{10}$. »

Dans la tribu des Winnebago (tribu Siou), les chefs de clans adressent à leurs confrères ${ }^{11}$, chefs des autres clans, des discours fort caractéristiques, modèles de cette étiquette 12 répandue dans toutes les civilisations des Indiens de l'Amérique du Nord. Chaque clan cuit des aliments, prépare du tabac pour les représentants des autres clans, lors de la fête de clan. Et voici par exemple des fragments des discours du chef du clan des Serpents 13 : « Je vous salue ; c'est bien ; comment pourrais-je dire autrement ? Je suis un pauvre homme sans valeur et vous vous êtes souvenus de moi. C'est bien... Vous avez pensé aux

8 ROTH, Games, But. Ethn. Queensland, p. 23, no 28.

9 Cette annonce du nom du clan survenant est un usage très général dans tout l'Est australien et se rattache au système de l'honneur et de la vertu du nom.

10 Fait notable, qui laisse à penser que se contractent alors des engagements matrimoniaux par la voie d'échanges de présents.

11 RADIN, Winnebago Tribe, XXXVIIth Annual Report of the Bureau of American Ethnology, p. 320 et sq.

12 V. art Etiquette, Handbook or American Indians, de HODGE.

13 p. 326, par exception, deux des chefs invités sont membres du clan du Serpent.

On peut comparer les discours exactement superposables d'une fête funéraire (tabac). Tlingit, SWANTON, Tlingit Myths and Texts (Bull. of Am. Ethn., no 39), p. 372 
esprits et vous êtes venus vous asseoir avec moi... Vos plats vont être bientôt remplis, je vous salue donc encore, vous, humains qui prenez la place des esprits, etc. » Et lorsque chacun des chefs a mangé et qu'on a fait les offrandes de tabac dans le feu, la formule finale expose l'effet moral de la fête et de toutes ses prestations : " Je vous remercie d'être venus occuper ce siège, je vous suis reconnaissant. Vous m'avez encouragé... Les bénédictions de vos grands-pères (qui ont eu des révélations et que vous incarnez), sont égales à celles des esprits. Il est bien que vous ayez pris part à ma fête. Ceci doit être, que nos anciens ont dit : " Votre vie est faible et vous ne pouvez être fortifié que par le « conseil des braves. » Vous m'avez conseillé... C'est de la vie pour moi. »

Ainsi, d'un bout à l'autre de l'évolution humaine, il n'y a pas deux sagesses. Qu'on adopte donc comme principe de notre vie ce qui a toujours été un principe et le sera toujours : sortir de soi, donner, librement et obligatoirement ; on ne risque pas de se tromper. Un beau proverbe maori le dit :

Ko Maru kai atu

Ko Maru kai mai

ka ngohe ngohe.

« Donne autant que tu prends, tout sera très bien ${ }^{14}$. »

14 Rev. TAYLOR, Te Ika a Maui, Old New Zealand, p. 1,10, prov. 42, traduit fort brièvement a give as well as take and all will be right », mais la traduction littérale est probablement la suivante : Autant Maru donne, autant Maru prend, et ceci est bien, bien. (Maru est le Dieu de la guerre et de la justice.) 


\section{II}

\section{CONCLUSIONS DE SOCIOLOGIE ÉCONOMIQUE ET D'ÉCONOMIE POLITIQUE}

$\underline{\text { Retour à la table des matières }}$

Ces faits n'éclairent pas seulement notre morale et n'aident pas seulement à diriger notre idéal ; de leur point de vue, on peut mieux analyser les faits économiques les plus généraux, et même cette analyse aide à entrevoir de meilleurs procédés de gestion applicables à nos sociétés.

À plusieurs reprises, on a vu combien toute cette économie de l'échange-don était loin de rentrer dans les cadres de l'économie soidisant naturelle, de l'utilitarisme. Tous ces phénomènes si considérables de la vie économique de tous ces peuples - disons, pour fixer les esprits, qu'ils sont bons représentants de la grande civilisation néolithique - et toutes ces survivances considérables de ces traditions, dans les sociétés proches de nous ou dans les usages des nôtres, échappent aux schèmes que donnent d'ordinaire lés rares économistes qui ont voulu comparer les diverses économies connues ${ }^{15}$. Nous ajoutons donc nos observations répétées à celles de $\mathrm{M}$. Malinowski qui a consacré tout un travail à " faire sauter » les doctrines courantes sur l'économie « primitive » 16 .

Voici une chaîne de faits bien solide:

La notion de valeur fonctionne dans ces sociétés ; des surplus très grands, absolument parlant, sont amassés ; ils sont dépensés souvent

15 M. BUCHER, Entstehung der Volkswirtschaft (3e éd.), p. 73, a vu ces phénomènes économiques, mais en a sous-estimé l'importance en les réduisant à l'hospitalité.

16 Argonauts, p. 167 sq. ; Primitive Economics, Economic Journal, mars 1921. V. la préface de J. G. Frazer à Malinowski, Arg. 
en pure perte, avec un luxe relativement énorme ${ }^{17}$ et qui n'a rien de mercantile; il y a des signes de richesse, des sortes de monnaies ${ }^{18}$, qui sont échangées. Mais toute cette économie très riche est encore pleine d'éléments religieux : la monnaie a encore son pouvoir magique et est encore liée au clan ou à l'individu ${ }^{19}$; les diverses activités économiques, par exemple le marché, sont imprégnées de rites et de mythes ; elles gardent un caractère cérémoniel, obligatoire, efficace 20 ; elles sont pleines de rites et de droits. À ce point de vue nous répondons déjà à la question que posait Durkheim à propos de l'origine religieuse de la notion de valeur économique ${ }^{21}$. Ces faits répondent aussi à une foule de questions concernant les formes et les raisons de ce qu'on appelle si mal l'échange, le « troc », la permutatio ${ }^{22}$ des choses utiles, qu'à la suite des prudents Latins, suivant eux-mêmes Aristote ${ }^{23}$, une économie historique met à l'origine de la division du travail. C'est bien autre chose que de l'utile, qui circule dans ces sociétés de tous genres, la plupart déjà assez éclairées. Les clans, les âges et, généralement, les sexes - à cause des multiples rapports auxquels les contacts donnent lieu - sont dans un état de perpétuelle effervescence économique et cette excitation est elle-même fort peu terre à terre ; elle est bien moins prosaïque que nos ventes et achats, que nos louages de service ou que nos jeux de Bourse.

17 Un des cas maximum que nous pouvons citer est celui du sacrifice des chiens chez les Chukchee (v. plus haut p. 55, no 2). Il arrive que les propriétaires des plus beaux chenils massacrent tous leurs équipages de traîneaux et sont obligés d'en racheter de nouveaux.

18 V. plus haut.

19 Cf. plus haut.

20 MALINOWSKI, Arg., p. 95. CI. Frazer, préface au livre de M. Malinowski.

21 Formes élémentaires de la vie religieuse, p. 598, no 2.

22 Digeste, XVIII, I; De Contr. Emt., 1. Paulus nous explique le grand débat entre prudents Romains pour savoir si la « permutatio » était une vente. Tout le passage est intéressant, même l'erreur que fait le savant juriste dans son interprétation d'Homère. Il, VII, 472 à 475 : [...] veut bien dire acheter, mais que les monnaies grecques c'étaient le bronze, le fer, les peaux, les vaches ellesmêmes et les esclaves, qui avaient tous des valeurs déterminées.

23 Pol., livre I, 1257 a, 10 sq. ; remarquer le mot [...], ibid., 25. 
Cependant, on peut encore aller plus loin que nous ne sommes parvenus jusqu'ici. On peut dissoudre, brasser, colorer et définir autrement les notions principales dont nous nous sommes servis. Les termes que nous avons employés: présent, cadeau, don, ne sont pas euxmêmes tout à fait exacts. Nous n'en trouvons pas d'autres, voilà tout. Ces concepts de droit et d'économie que nous nous plaisons à opposer : liberté et obligation ; libéralité, générosité, luxe et épargne, intérêt, utilité, il serait bon de les remettre au creuset. Nous ne pouvons donner que des indications à ce sujet : choisissons par exemple ${ }^{24}$ les Trobriand. C'est encore une notion complexe qui inspire tous les actes économiques que nous avons décrits ; et cette notion n'est ni celle de la prestation purement libre et purement gratuite, ni celle de la production et de l'échange purement intéressés de l'utile. C'est une sorte d'hybride qui a fleuri là-bas.

M. Malinowski a fait un effort sérieux ${ }^{25}$ pour classer du point de vue des mobiles, de l'intérêt et du désintéressement, toutes les transactions qu'il constate chez ses Trobriandais ; il les étage entre le don pur et le troc pur après marchandage 26 . Cette classification est au fond inapplicable. Ainsi, selon M. Malinowski, le type du don pur serait le don entre époux ${ }^{27}$. Or, précisément, à notre sens, l'un des faits les plus importants signalés par $\mathrm{M}$. Malinowski et qui jette une lumière éclatante sur tous les rapports sexuels dans toute l'humanité, consiste à rapprocher le mapula 28 , le paiement « constant » de l'homme à sa femme, d'une sorte de salaire pour service sexuel rendu ${ }^{29}$. De même les cadeaux au chef sont des tributs; les distributions de nourriture

24 Nous pourrions tout aussi bien choisir la sadaqa arabe; aumône, prix de la fiancée, justice, impôt. Cf. plus haut.

25 Argonauts, p. 177.

26 Il est très remarquable que, dans ce cas, il n'y ait pas vente, car Il n'y a as échange de vaygu'a, de monnaies. Le maximum d'économie auquel se sont haussés les Trobriandais, ne va donc pas jusqu'à l'usage de la monnaie dans l'échange lui-même.

27 Pure gift.

28 Ibid.

29 Le mot s'applique au paiement de la sorte de prostitution licite des filles non mariées; cf. Arg., p. 183. 
(sagali) sont des indemnités pour travaux, pour rites accomplis, par exemple en cas de veillée funéraire ${ }^{30}$. Au fond, de même que ces dons ne sont pas libres, ils ne sont pas réellement désintéressés. Ce sont déjà des contre-prestations pour la plupart, et faites même en vue non seulement de payer des services et des choses, mais aussi de maintenir une alliance profitable 31 et qui ne peut même être refusée, comme par exemple l'alliance entre tribus de pêcheurs 32 et tribus d'agriculteurs ou de potiers. Or, ce fait est général, nous l'avons rencontré par exemple en pays Maori, Tsimshian ${ }^{33}$, etc. On voit donc où réside cette force, à la fois mystique et pratique qui soude les clans et en même temps les divise, qui divise leur travail et en même temps les contraint à l'échange. Même dans ces sociétés, l'individu et le groupe, ou plutôt le sous-groupe, se sont toujours senti le droit souverain de refuser le contrat : c'est ce qui donne un aspect de générosité à cette circulation des biens ; mais, d'autre part, ils n'avaient à ce refus, normalement, ni droit ni intérêt ; et c'est ce qui rend ces lointaines sociétés tout de même parentes des nôtres.

L'emploi de la monnaie pourrait suggérer d'autres réflexions. Les vaygu'a des Trobriand, bracelets et colliers, tout comme les cuivres du Nord-Ouest américain ou les wampun iroquois, sont à la fois des richesses, des signes ${ }^{34}$ de richesse, des moyens d'échange et de paie-

30 Cf. plus haut. Le mot sagali (cf. hakari) veut dire distribution.

31 Cf. plus haut; en particulier le don de I'urigubu au beau-frère : produits de récolte en échange de travail.

32 V. plus haut (wasi).

33 Maori, v. plus haut. La division du travail (et la façon dont elle fonctionne en vue de la fête entre clans Tsimshian), est admirablement décrite dans un mythe de potlatch, Boas, Tsimshian Mythology, XXXIst Ann, Rep. Bur. Am. Ethn., pp. 274, 275 ; cf. p. 378. Des exemples de ce genre pourraient être indéfiniment multipliés. Ces institutions économiques existent en effet, même chez les sociétés infiniment moins évoluées. V. par exemple en Australie la remarquable position d'un groupe local possesseur d'un gisement d'ocre rouge (AISTON et HORNE, Savage Life in Central Australia, Londres, 1924, pp. 81, 130).

$34 \mathrm{~V}$. plus haut. L'équivalence dans les langues germaniques des mots token et zeichen, pour désigner la monnaie en général, garde la trace de ces institutions. le signe qu'est la monnaie, le signe qu'elle porte et le gage qu'elle est sont une seule et même chose - comme la signature d'un homme est encore ce qui engage sa responsabilité. 
ment, et aussi des choses qu'il faut donner, voire détruire. Seulement, ce sont encore des gages liés aux personnes qui les emploient, et ces gages les lient. Mais comme, d'autre part, ils servent déjà de signes monétaires, on a intérêt à les donner pour pouvoir en posséder d'autres à nouveau, en les transformant en marchandises ou en services qui se retransformeront à leur tour en monnaies. On dirait vraiment que le chef trobriandais ou tsimshian procède à un lointain degré à la façon du capitaliste qui sait se défaire de sa monnaie en temps utile, pour reconstituer ensuite son capital mobile. Intérêt et désintéressement expliquent également cette forme de la circulation des richesses et celle de la circulation archaïque des signes de richesse qui les suivent.

Même la destruction pure des richesses ne correspond pas à ce détachement complet qu'on croirait y trouver. Même ces actes de grandeur ne sont pas exempts d'égotisme. La forme purement somptuaire, presque toujours exagérée, souvent purement destructrice, de la consommation, où des biens considérables et longtemps amassés sont donnés tout d'un coup ou même détruits, surtout en cas de potlatch ${ }^{35}$, donne à ces institutions un air de pure dépense dispendieuse, de prodigalité enfantine. En effet, et en fait, non seulement on y fait disparaître des choses utiles, de riches aliments consommés avec excès, mais même on y détruit pour le plaisir de détruire, par exemple, ces cuivres, ces monnaies, que les chefs tsimshian, tlingit et haïda jettent à l'eau et que brisent les chefs kwakiultl et ceux des tribus qui leur sont alliées. Mais le motif de ces dons et de ces consommations forcenées, de ces pertes et de ces destructions folles de richesses, n'est, à aucun degré, surtout dans les sociétés à potlatch, désintéressé. Entre chefs et vassaux, entre vassaux et tenants, par ces dons, c'est la hiérarchie qui s'établit. Donner, c'est manifester sa supériorité, être plus, plus haut, magister ; accepter sans rendre ou sans rendre plus, c'est se subordonner, devenir client et serviteur, devenir petit, choir plus bas (minister).

35 V. Davy, Foi jurée, p. 344 sq. ; M. Davy (Des clans aux Empires; Éléments de Sociologie, I) a seulement exagéré l'importance de ces faits. Le potlatch est utile pour établir la hiérarchie et l'établit souvent, mais il n'y est pas absolument nécessaire. Ainsi les sociétés africaines, nigritiennes ou bantu, ou n'ont pas le potlatch, ou n'en ont en tout cas pas de très développé, ou peut-être l'ont perdu - et elles ont toutes les formes d'organisation politique possibles. 
Le rituel magique du kula appelé mwasila ${ }^{36}$ est plein de formules et de symboles qui démontrent que le futur contractant recherche avant tout ce profit : la supériorité sociale, et on pourrait même dire brutale. Ainsi, après avoir enchanté la noix de bétel dont ils vont se servir avec leurs partenaires, après avoir enchanté le chef, ses camarades, leurs porcs, les colliers, puis la tête et ses " ouvertures », plus tout ce qu'on apporte, les pari, dons d'ouverture, etc., après avoir enchanté tout cela, le magicien chante, non sans exagération ${ }^{37}$ :

Je renverse la montagne, la montagne bouge, la montagne s'écroule, etc. Mon charme va au sommet de la montagne de Dobu... Mon canot va couler.... etc. Ma renommée est comme le tonnerre ; mon pas est comme le bruit que font les sorciers volants. Tudududu.

Être le premier, le plus beau, le plus chanceux, le plus fort et le plus riche, voilà ce qu'on cherche et comment on l'obtient. Plus tard, le chef confirme son mana en redistribuant à ses vassaux, parents, ce qu'il vient de recevoir ; il maintient son rang parmi les chefs en rendant bracelets contre colliers, hospitalité contre visites, et ainsi de suite... Dans ce cas la richesse est, à tout point de vue, autant un moyen de prestige qu'une chose d'utilité. Mais est-il sûr qu'il en soit autrement parmi nous et que même chez nous la richesse ne soit pas avant tout le moyen de commander aux hommes?

Passons maintenant au feu d'épreuve l'autre notion que nous venons d'opposer à celle de don et de désintéressement : la notion d'intérêt, de recherche individuelle de l'utile. Celle-là non plus ne se présente pas comme elle fonctionne dans notre esprit à nous. Si quelque motif équivalent anime chefs trobriandais ou américains, clans andamans, etc., ou animait autrefois généreux Hindous, nobles Germains et Celtes dans leurs dons et dépenses, ce n'est pas la froide raison du

Arg., pp. 199 à 201 ; cf. p. 203.

37 Ibid., p. 199. Le mot montagne désigne, dans cette poésie, les îles d'Entrecasteaux. Le canot coulera sous le poids des marchandises rapportées du kula. Cf. autre formule, p. 200, texte avec commentaires, p. 441 ; cf. p. 442, remarquable jeu de mots sur « écumer ». Cf. formule, p. 205 ; cf. plus haut p. 124, no 1. 
marchand, du banquier et du capitaliste. Dans ces civilisations, on est intéressé, mais d'autre façon que de notre temps. On thésaurise, mais pour dépenser, pour " obliger», pour avoir des "hommes liges». D'autre part, on échange, mais ce sont surtout des choses de luxe, des ornements, des vêtements, ou ce sont des choses immédiatement consommées, des festins. On rend avec usure, mais c'est pour humilier le premier donateur ou échangiste et non pas seulement pour le récompenser de la perte que lui cause une " consommation différée ». Il y a intérêt, mais cet intérêt n'est qu'analogue à celui qui, dit-on, nous guide.

Entre l'économie relativement amorphe et désintéressée, à l'intérieur des sous-groupes, qui règle la vie des clans australiens ou américains du Nord (Est et Prairie), d'une part ; et l'économie individuelle et du pur intérêt que nos sociétés ont connu au moins en partie, dès qu'elle fut trouvée par les populations sémitiques et grecques, d'autre part ; entre ces deux types, dis-je, s'est étagée toute une série immense d'institutions et d'événements économiques, et cette série n'est pas gouvernée par le rationalisme économique dont on fait si volontiers la théorie.

Le mot même d'intérêt est récent, d'origine technique comptable : « interest », latin, qu'on écrivait sur les livres de comptes, en face des rentes à percevoir. Dans les morales anciennes les plus épicuriennes, c'est le bien et le plaisir qu'on recherche, et non pas la matérielle utilité. Il a fallu la victoire du rationalisme et du mercantilisme pour que soient mises en vigueur, et élevées à la hauteur de principes, les notions de profit et d'individu. On peut presque dater - après Mandeville (Fable des Abeilles) - le triomphe de la notion d'intérêt individuel. On ne peut que difficilement et seulement par périphrase traduire ces derniers mots, en latin ou en grec, ou en arabe. Même les hommes qui écrivirent le sanskrit classique, qui employèrent le mot artha, assez proche de notre idée d'intérêt, se sont fait de l'intérêt, comme des autres catégories de l'action, une autre idée que nous. Les livres sacrés de l'Inde classique répartissent déjà les activités humaines suivant : la loi (dharma), l'intérêt (artha), le désir (kama). Mais c'est avant tout de l'intérêt politique qu'il s'agit : celui du roi et des brahmanes, des ministres, celui du royaume et de chaque caste. La littérature considérable des Nitiçastra n'est pas économique. 
Ce sont nos sociétés d'Occident qui ont, très récemment, fait de l'homme un " animal économique ». Mais nous ne sommes pas encore tous des êtres de ce genre. Dans nos masses et dans nos élites, la dépense pure et irrationnelle est de pratique courante ; elle est encore caractéristique des quelques fossiles de notre noblesse. L'homo oeconomicus n'est pas derrière nous, il est devant nous; comme l'homme de la morale et du devoir; comme l'homme de la science et de la raison. L'homme a été très longtemps autre chose ; et il n'y a pas bien longtemps qu'il est une machine, compliquée d'une machine à calculer.

D'ailleurs nous sommes encore heureusement éloigné de ce constant et glacial calcul utilitaire. Qu'on analyse de façon approfondie, statistique, comme M. Halbwachs l'a fait pour les classes ouvrières, ce qu'est notre consommation, notre dépense à nous, occidentaux des classes moyennes. Combien de besoins satisfaisons-nous ? et combien de tendances ne satisfaisons-nous pas qui n'ont pas pour but dernier l'utile ? L'homme riche, lui, combien affecte-il, combien peut-il affecter de son revenu à son utilité personnelle ? Ses dépenses de luxe, d'art, de folie, de serviteurs ne le font-elles pas ressembler aux nobles d'autrefois ou aux chefs barbares dont nous avons décrit les mœurs?

Est-il bien qu'il en soit ainsi ? C'est une autre question. Il est bon peut-être qu'il y ait d'autres moyens de dépenser et d'échanger que la pure dépense. Cependant, à notre sens, ce n'est pas dans le calcul des besoins individuels qu'on trouvera la méthode de la meilleure économie. Nous devons, je le crois, même en tant que nous voulons développer notre propre richesse, rester autre chose que de purs financiers, tout en devenant de meilleurs comptables et de meilleurs gestionnaires. La poursuite brutale des fins de l'individu est nuisible aux fins et à la paix de l'ensemble, au rythme de son travail et de ses joies et - par l'effet en retour - à l'individu lui-même.

Déjà, nous venons de le voir, des sections importantes, des associations de nos entreprises capitalistes elles-mêmes, cherchent en groupes à s'attacher leurs employés en groupes. D'autre part, tous les groupements syndicalistes, ceux des patrons comme ceux des salariés, prétendent qu'ils défendent et représentent l'intérêt général avec autant de 
ferveur que l'intérêt particulier de leurs adhérents ou même de leurs corporations. Ces beaux discours sont, il est vrai, émaillés de bien des métaphores. Cependant, il faut le constater, non seulement la morale et la philosophie, mais même encore l'opinion et l'art économique luimême, commencent à se hausser à ce niveau " social ». On sent qu'on ne peut plus bien faire travailler que des hommes sûrs d'être loyalement payés toute leur vie, du travail qu'ils ont loyalement exécuté, en même temps pour autrui que pour eux-mêmes. Le producteur échangiste sent de nouveau - il a toujours senti - mais cette fois, il sent de façon aiguë, qu'il échange plus qu'un produit ou qu'un temps de travail, qu'il donne quelque chose de soi ; son temps, sa vie, Il veut donc être récompensé, même avec modération, de ce don. Et lui refuser cette récompense c'est l'inciter à la paresse et au moindre rendement.

Peut-être pourrions-nous indiquer une conclusion à la fois sociologique et pratique. La fameuse Sourate LXIV, « déception mutuelle » (Jugement dernier), donnée à La Mecque, à Mahomet, dit de Dieu :

15. Vos richesses et vos enfants sont votre tentation pendant que Dieu tient en réserve une récompense magnifique.

16. Craignez Dieu de toutes vos forces; écoutez, obéissez, faites l'aumône (sadaqa) dans votre propre intérêt. Celui qui se tient en garde contre son avarice sera heureux.

17. Si vous faites à Dieu un prêt généreux, il vous paiera le double, il vous pardonnera car il est reconnaissant et plein de longanimité.

18. Il connaît les choses visibles et invisibles, il est le puissant et le sage.

Remplacez le nom d'Allah par celui de la société et celui du groupe professionnel ou additionnez les trois noms, si vous êtes religieux ; remplacez le concept d'aumône par celui de coopération, d'un travail, d'une prestation faite en vue d'autrui : vous aurez une assez bonne idée de l'art économique qui est en voie d'enfantement laborieux. On le voit déjà fonctionner dans certains groupements économiques, et dans les cœurs des masses qui ont, bien souvent, mieux que leurs dirigeants, le sens de leurs intérêts, de l'intérêt commun. 
Peut-être, en étudiant ces côtés obscurs de la vie sociale, arrivera-ton à éclairer un peu la route que doivent prendre nos nations, leur morale en même temps que leur économie.

\section{III}

\section{CONCLUSION DE SOCIOLOGIE GÉNÉRALE ET DE MORALE}

\section{$\underline{\text { Retour à la table des matières }}$}

Qu'on nous permette encore une remarque de méthode à propos de celle que nous avons suivie.

Non pas que nous voulions proposer ce travail comme un modèle. Il est tout d'indications. Il est insuffisamment complet et l'analyse pourrait encore être poussée plus loin ${ }^{38}$. Au fond, ce sont plutôt des questions que nous posons aux historiens, aux ethnographes, ce sont des objets d'enquêtes que nous proposons plutôt que nous ne résolvons un problème et ne rendons une réponse définitive. Il nous suffit pour le moment d'être persuadé que, dans cette direction, on trouvera de nombreux faits,

Mais, s'il en est ainsi, c'est qu'il y a dans cette façon de traiter un problème un principe heuristique que nous voudrions dégager. Les faits que nous avons étudiés sont tous, qu'on nous permette l'expres-

38 L'aire sur laquelle nos recherches eussent dû porter le plus avec celles que nous avons étudiées, est la Micronésie. Il y existe un système de monnaie et de contrats extrêmement important, surtout à Yap et aux Palaos. En Indochine, surtout parmi les Mon-Khmer, en Assam et chez les Thibéto-Birmans, il y a aussi des institutions de ce genre. Enfin les Berbères ont développé les remarquables usages de la [haoussa (V. WESTERMARCK, Marriage Ceremonies in Morocco. V. ind. s. v. Present). MM. Doutté et Maunier, plus compétents que nous, se sont réservé l'étude de ce fait. Le vieux droit sémitique comme la coutume bédouine donneront aussi de précieux documents. 
sion, des faits sociaux totaux ou, si l'on veut - mais nous aimons moins le mot -généraux : c'est-à-dire qu'ils mettent en branle dans certains cas la totalité de la société et de ses institutions (potlatch, clans affrontés, tribus se visitant, etc.) et dans d'autres cas, seulement un très grand nombre d'institutions, en particulier lorsque ces échanges et ces contrats concernent plutôt des individus.

Tous ces phénomènes sont à la fois juridiques, économiques, religieux, et même esthétiques, morphologiques, etc. Ils sont juridiques, de droit privé et public, de moralité organisée et diffuse, strictement obligatoires ou simplement loués et blâmés, politiques et domestiques en même temps, intéressant les classes sociales aussi bien que les clans et les familles. Ils sont religieux : de religion stricte et de magie et d'animisme et de mentalité religieuse diffuse. Ils sont économiques: car l'idée de la valeur, de l'utile, de l'intérêt, du luxe, de la richesse, de l'acquisition de l'accumulation, et d'autre part, celle de la consommation, même celle de la dépense pure, purement somptuaire, y sont partout présentes, bien qu'elles y soient entendues autrement qu'aujourd'hui chez nous. D'autre part, ces institutions ont un côté esthétique important dont nous avons fait délibérément abstraction dans cette étude : mais les danses qu'on exécute alternativement, les chants et les parades de toutes sortes, les représentations dramatiques qu'on se donne de camp à camp et d'associé à associé ; les objets de toutes sortes qu'on fabrique, use, orne, polit, recueille et transmet avec amour, tout ce qu'on reçoit avec joie et présente avec succès, les festins euxmêmes auxquels tous participent ; tout, nourriture, objets et services, même le " respect », comme disent les Tlingit, tout est cause d'émotion esthétique et non pas seulement d'émotions de l'ordre du moral ou de l'intérêt ${ }^{39}$. Ceci est vrai non seulement de la Mélanésie, mais encore plus particulièrement de ce système qu'est le potlatch du NordOuest américain, encore plus vrai de la fête-marché du monde indo-

39 V. le « rituel de Beauté » dans le « Kula » des Trobriand, MALINOWSKI, p. 334 et suivantes, 336, " notre partenaire nous voit, voit que notre figure est belle, il nous jette ses vaygu'a ». Cf. THURNWALD sur l'usage de l'argent comme ornement, Forschungen, Ill, p. 39; cf. l'expression Prachtbaurn, tome III, p. 144, v. 6, v. 13; 156, v. 12; pour désigner un homme ou une femme décorés de monnaie. Ailleurs le chef est désigné comme l' « arbre », I, p. 298, v. 3. Ailleurs l'homme décoré dégage un parfum, I, p. 192, v. 7; v. 13, 14. 
européen ${ }^{40}$. Enfin, ce sont clairement des phénomènes morphologiques. Tout s'y passe au cours d'assemblées, de foires et de marchés, ou tout au moins de fêtes qui en tiennent lieu. Toutes celles-ci supposent des congrégations dont la permanence peut excéder une saison de concentration sociale, comme les potlatch d'hiver des Kwakiutl, ou des semaines, comme les expéditions maritimes des Mélanésiens. D'autre part, il faut qu'il y ait des routes, des pistes tout au moins, des mers ou des lacs où on puisse se transporter en paix. Il faut les alliances tribales et intertribales ou internationales, le commercium et le connubium ${ }^{41}$.

Ce sont donc plus que des thèmes, plus que des éléments d'institutions, plus que des institutions complexes, plus même que des systèmes d'institutions divisés par exemple en religion, droit, économie, etc. Ce sont des «touts ", des systèmes sociaux entiers dont nous avons essayé de décrire le fonctionnement. Nous avons vu des sociétés à l'état dynamique ou physiologique. Nous ne les avons pas étudiées comme si elles étaient figées, dans un état statique ou plutôt cadavérique, et encore moins les avons-nous décomposées et disséquées en règles de droit, en mythes, en valeurs et en prix. C'est en considérant le tout ensemble que nous avons pu percevoir l'essentiel, le mouvement du tout, l'aspect vivant, l'instant fugitif où la société prend, où les hommes prennent conscience sentimentale d'eux-mêmes et de leur situation vis-à-vis d'autrui. Il y a, dans cette observation concrète de la vie sociale, le moyen de trouver des faits nouveaux que nous commençons seulement à entrevoir. Rien à notre avis n'est plus urgent ni fructueux que cette étude des faits sociaux.

Elle a un double avantage. D'abord un avantage de généralité, car ces faits de fonctionnement général ont des chances d'être plus universels que les diverses institutions ou que les divers thèmes de ces institutions, toujours plus ou moins accidentellement teintés d'une couleur locale. Mais surtout, elle a un avantage de réalité. On arrive ainsi à voir les choses sociales elles-mêmes, dans le concret, comme elles sont. Dans les sociétés, on saisit plus que des idées ou des règles, on saisit des hommes, des groupes et leurs comportements. On les voit se

40 Marchés aux fiancées; notion de fête, feria foire.

41 Cf. THURNWALD, ibid., III, p. 36. 
mouvoir comme en mécanique on voit des masses et des systèmes, ou comme dans la mer nous voyons des pieuvres et des anémones. Nous apercevons des nombres d'hommes, des forces mobiles, et qui flottent dans leur milieu et dans leurs sentiments.

Les historiens sentent et objectent à juste titre que les sociologues font trop d'abstractions et séparent trop les divers éléments des sociétés les uns des autres. Il faut faire comme eux : observer ce qui est donné. Or, le donné, c'est Rome, c'est Athènes, c'est le Français moyen, c'est le Mélanésien de telle ou telle île, et non pas la prière ou le droit en soi. Après avoir forcément un peu trop divisé et abstrait, il faut que les sociologues s'efforcent de recomposer le tout. Ils trouveront ainsi de fécondes données. - Ils trouveront aussi le moyen de satisfaire les psychologues. Ceux-ci sentent vivement leur privilège, et surtout les psycho-pathologistes ont la certitude d'étudier du concret. Tous étudient ou devraient observer le comportement d'êtres totaux et non divisés en facultés. Il faut les imiter. L'étude du concret, qui est du complet, est possible et plus captivante et plus explicative encore en sociologie. Nous, nous observons des réactions complètes et complexes de quantités numériquement définies d'hommes, d'êtres complets et complexes. Nous aussi, nous décrivons ce qu'ils sont dans leurs organismes et leurs psychai, en même temps que nous décrivons ce comportement de cette masse et les psychoses qui y correspondent : sentiments, idées, volitions de la foule ou des sociétés organisées et de leurs sous-groupes. Nous aussi, nous voyons des corps et les réactions de ces corps, dont idées et sentiments sont d'ordinaire les interprétations et, plus rarement, les motifs. Le principe et la fin de la sociologie, c'est d'apercevoir le groupe entier et son comportement tout entier.

Nous n'avons pas eu le temps - ç'aurait été indûment étendre un sujet restreint - d'essayer d'apercevoir dès maintenant le tréfonds morphologique de tous les faits que nous avons indiqués. Il est peut-être cependant utile d'indiquer, au moins à titre d'exemple de la méthode que nous voudrions suivre, dans quelle voie nous poursuivrions cette recherche.

Toutes les sociétés que nous avons décrites ci-dessus, sauf nos sociétés européennes, sont des sociétés segmentées. Même les sociétés 
indo-européennes, la romaine d'avant les Douze Tables, les sociétés germaniques encore très tard, jusqu'à la rédaction de l'Edda, la société irlandaise jusqu'à la rédaction de sa principale littérature étaient encore à base de clans et tout au moins de grandes familles plus ou moins indivises à l'intérieur et plus ou moins isolées les unes des autres à l'extérieur. Toutes ces sociétés sont, ou étaient, loin de notre unification et de l'unité qu'une histoire insuffisante leur prête. D'autre part, à l'intérieur de ces groupes, les individus, même fortement marqués, étaient moins tristes, moins sérieux, moins avares et moins personnels que nous ne sommes; extérieurement tout au moins, ils étaient ou sont plus généreux, plus donnants que nous. Lorsque, lors des fêtes tribales, des cérémonies des clans affrontés et des familles qui s'allient ou s'initient réciproquement, les groupes se rendent visite ; même lorsque, dans des sociétés plus avancées - quand la loi « d'hospitalité » s'est développée - la loi des amitiés et des contrats avec les dieux, est venue assurer la «paix » des "marchés » et des villes ; pendant tout un temps considérable et dans un nombre considérable de sociétés, les hommes se sont abordés dans un curieux état d'esprit, de crainte et d'hostilité exagérées et de générosité également exagérée, mais qui ne sont folles qu'à nos yeux. Dans toutes les sociétés qui nous ont précédés immédiatement et encore nous entourent, et même dans de nombreux usages de notre moralité populaire, il n'y a pas de milieu : se confier entièrement ou se défier entièrement déposer ses armes et renoncer à sa magie, ou donner tout depuis l'hospitalité fugace jusqu'aux filles et aux biens. C'est dans des états de ce genre que les hommes ont renoncé, à leur quant-à-soi et ont su s'engager à donner et à rendre.

C'est qu'ils n'avaient pas le choix. Deux groupes d'hommes qui se rencontrent ne peuvent que : ou s'écarter - et, s'ils se marquent une méfiance ou se lancent un défi, se battre - ou bien traiter. Jusqu'à des droits très proches de nous, jusqu'à des économies pas très éloignées de la nôtre, ce sont toujours des étrangers avec lesquels on " traite », même quand on est allié. Les gens de Kiriwina dans les Trobriand dirent à M. Malinowski 42 : "Les hommes de Dobu ne sont pas bons comme nous ; ils sont cruels, ils sont cannibales ; quand nous arrivons à Dobu, nous les craignons. Ils pourraient nous tuer. Mais voilà, je

42 Argonauts, p. 246. 
crache de la racine de gingembre, et leur esprit change. Ils déposent leurs lances et nous reçoivent bien. » Rien ne traduit mieux cette instabilité entre la fête et la guerre.

Un des meilleurs ethnographes, M. Thurnwald, nous décrit, à propos d'une autre tribu de Mélanésie, dans une statistique généalogique ${ }^{43}$, un événement précis qui montre également bien comment ces gens passent, en groupe et d'un coup, de la fête à la bataille. Buleau, un chef, avait invité Bobal, un autre chef et ses gens à un festin, probablement le premier d'une longue série. On commença à répéter les danses, pendant toute une nuit. Au matin, tous étaient excités par la nuit de veille, de danses et de chants. Sur une simple observation de Buleau, un des hommes de Bobal le tua. Et la troupe massacra, pilla et enleva les femmes du village. «Buleau et Bobal étaient plutôt amis et seulement rivaux ", a-t-on dit à $\mathrm{M}$. Thurnwald. Nous avons tous observé de ces faits, même encore autour de nous.

C'est en opposant la raison et le sentiment, c'est en posant la volonté de paix contre de brusques folies de ce genre que les peuples réussissent à substituer l'alliance, le don et le commerce à la guerre et à l'isolement et à la stagnation.

Voilà donc ce que l'on trouverait au bout de ces recherches. Les sociétés ont progressé dans la mesure où elles-mêmes, leurs sousgroupes et enfin leurs individus, ont su stabiliser leurs rapports, donner, recevoir, et enfin, rendre. Pour commercer, il fallut d'abord savoir poser les lances. C'est alors qu'on a réussi à échanger les biens et les personnes, non plus seulement de clans à clans, mais de tribus à tribus et de nations à nations et - surtout - d'individus à individus. C'est seulement ensuite que les gens ont su se créer, se satisfaire mutuellement des intérêts, et enfin, les défendre sans avoir à recourir aux armes. C'est ainsi que le clan, la tribu, les peuples ont su - et c'est ainsi que demain, dans notre monde dit civilisé, les classes et les nations et aussi les individus, doivent savoir - s'opposer sans se massacrer et se donner sans se sacrifier les uns aux autres. C'est là un des secrets permanents de leur sagesse et de leur solidarité.

43 Salomo Inseln, tome III, table 85, note 2. 
Il n'y a pas d'autre morale, ni d'autre économie, ni d'autres pratiques sociales que celles-là. Les Bretons, les Chroniques d'Arthur, racontent ${ }^{4}$ comment le roi Arthur, avec l'aide d'un charpentier de Cornouailles inventa cette merveille de sa cour : la « Table Ronde » miraculeuse autour de laquelle les chevaliers ne se battirent plus. Auparavant, " par sordide envie ", dans des échauffourées stupides, des duels et des meurtres ensanglantaient les plus beaux festins. Le charpentier dit à Arthur : " Je te ferai une table très belle, où ils pourront s'asseoir seize cents et plus, et tourner autour, et dont personne ne sera exclu... Aucun chevalier ne pourra livrer combat, car là, le haut placé sera sur le même pied que le bas placé. » Il n'y eut plus de « haut bout » et partant, plus de querelles. Partout où Arthur transporta sa Table, joyeuse et invincible resta sa noble compagnie. C'est ainsi qu'aujourd'hui encore se font les nations, fortes et riches, heureuses et bonnes. Les peuples, les classes, les familles, les individus, pourront s'enrichir, ils ne seront heureux que quand ils sauront s'asseoir, tels des chevaliers, autour de la richesse commune. Il est inutile d'aller chercher bien loin quel est le bien et le bonheur. Il est là, dans la paix imposée, dans le travail bien rythmé, en commun et solitaire alternativement, dans la richesse amassée puis redistribuée dans le respect mutuel et la générosité réciproque que l'éducation enseigne.

On voit comment on peut étudier, dans certains cas, le comportement humain total, la vie sociale tout entière ; et on voit aussi comment cette étude concrète peut mener non seulement à une science des mœurs, à une science sociale partielle, mais même à des conclusions de morale, ou plutôt - pour reprendre le vieux mot - de " civilité ", de " civisme ", comme on dit maintenant. Des études de ce genre permettent en effet d'entrevoir, de mesurer, de balancer les divers mobiles esthétiques, moraux, religieux, économiques, les divers facteurs matériels et démographiques dont l'ensemble fonde la société et constitue la vie en commun, et dont la direction consciente est l'art suprême, la Politique, au sens socratique du mot.

44 Layamon's Brut, vers 22736 sq.; Brut, vers 9994 sq. 


\title{
Annexe 5
}

\author{
Marcel Mauss \\ Épinal, 10 mai 1872 - Paris, 1er février 1950 \\ Par Marcel Fournier \\ professeur titulaire du département de sociologie \\ de l'université de Montréal
}

$\underline{\text { Retour à la table des matières }}$

Père de l'ethnographie française, Marcel Mauss a exercé une influence profonde sur les sciences sociales et humaines et a légué un héritage intellectuel d'une richesse inépuisable. Spontanément, on l'identifie à Émile Durkheim (18541917), son oncle maternel et son maître. Né dans une famille de négociants et de rabbins, Mauss, après une agrégation de philosophie (1895), s'oriente vers la sociologie religieuse et acquiert, à l'École pratique des hautes études, de solides connaissances en philologie, en histoire des religions et aussi en ethnologie.

Son premier grand travail, réalisé en collaboration avec Henri Hubert (18721927), porte sur " la nature et la fonction du sacrifice " (1899). L'essai paraît dans L'Année sociologique que Durkheim vient de fonder et dont Mauss est l'un des plus actifs collaborateurs. À l'EPHE, il est chargé, en 1901, de l'enseignement de "l'Histoire des religions des peuples non-civilisés". Les recherches qu'il entreprend ont pour objet les manifestations rituelles de la vie religieuse et pour objectif l'élaboration d'une théorie du sacré, mais s'élargissent bientôt pour toucher à la théorie de la connaissance. La Grande guerre, que Mauss, engagé volontaire, effectue comme interprète, emporte Durkheim, son fils André et plusieurs collaborateurs de L'Année sociologique. Après l'Armistice, Mauss prend la relève, relance la revue et, en collaboration avec Lucien Lévi-Bruhl et Paul Rivet, fonde en 1925 l'Institut d'ethnologie de Paris, autour duquel se constitue une véritable école qui organise, en Afrique surtout, les premières grandes expéditions ethnologiques. Il est élu en 1930 au Collège de France (chaire de sociologie).

Parmi ses écrits, le plus fameux est son Essai sur le don (1925). Marcel Mauss est un savant, mais c'est aussi un militant. Impliqué très tôt dans l'action politique, du côté des dreyfusards et des socialistes, il collabore au Mouvement social et 
participe à la mise sur pied de la Société nouvelle de librairie et d'édition. Une fois professeur, il poursuit ses activités au sein du mouvement coopératif et du parti socialiste et publie de nombreux articles dans l'Humanité, dont il est l'un des fondateurs. Après la guerre, il entreprend la rédaction d'un grand ouvrage sur la Nation et après avoir publié ses "Observations sur la violence" dans la Vie socialiste, élabore le plan d'un livre sur le bolchevisme.

La grande force de Mauss est sa capacité de s'adapter aux réalités nouvelles tout en demeurant fidèle à ses convictions (et à son ami Jaurès) : il critique le bolchevisme sans renier son adhésion au socialisme ; il s'intéresse à la question nationale tout en demeurant internationaliste ; enfin, comme d'autres pacifistes, il veut éviter la guerre, mais il est le premier à dénoncer le fascisme. Ses Écrits politiques comprennent de précieuses "appréciations" où se mêlent l'ardeur du savant et celle du militant.

Marcel Fournier

professeur titulaire du département de sociologie de l'université de Montréal

\section{Fin du texte}

